\title{
Design of Science Orbits About Planetary Satellites: Application to Europa
}

\author{
Marci E. Paskowitz* and Daniel J. Scheeres ${ }^{\dagger}$ \\ University of Michigan, Ann Arbor, Michigan 48109
}

DOI: $10.2514 / 1.19464$

\begin{abstract}
Low-altitude, near-polar orbits are very desirable as science orbits for missions to planetary satellites, such as Jupiter's moon Europa. However, previous research has shown that a spacecraft in such a low-altitude, near-polar orbit about Europa will impact relatively quickly. To remedy this we study the underlying dynamics governing motion in the vicinity of Europa using a model that includes the tidal force from Jupiter and the $J_{2}, C_{22}$, and $J_{3}$ gravity coefficients of Europa. Averaging is applied twice to reduce the original 3-degree-of-freedom system to an integrable 1-degree-of-freedom system. By studying this simplified 1-degree-of-freedom system we can identify paths that yield long lifetime trajectories. In order for a spacecraft in the original 3-degree-of-freedom system to follow one of these paths, initial conditions must be computed such that the trajectory in the 3-degree-of-freedom system follows the trajectory in the 1-degree-of-freedom system on average. To ensure this we develop a technique that allows us to systematically compute the necessary initial conditions in the 3-degree-of-freedom system to maximize the orbit lifetime. The resulting orbits have lifetimes on the order of 150 days, compared with lifetimes on the order of 40 days for randomly chosen initial conditions.
\end{abstract}

\section{Introduction}

$\mathbf{T}$ HE exploration of planetary satellites is currently an active area of research. Missions have been proposed to study the Galilean satellites of Jupiter, in particular, Europa, Ganymede, and Callisto. Of these, interest in Europa is the most intense because Europa may have the conditions necessary to sustain life. When designing a science orbit for a mission to Europa or another planetary satellite, the largest consideration is the science requirements of the mission, which can include imaging the surface, determining its composition, and measuring features of the planetary satellite such as the gravity field and the radiation environment. For this reason, the best science orbits will have low altitudes and near-polar inclinations. However, previous research [1] has shown that these types of orbits about Europa are unstable. Consider Fig. 1, which shows the eccentricity as a function of time for a few trajectories. These trajectories have an initial altitude of $100 \mathrm{~km}$, an inclination of $70 \mathrm{deg}$, and different initial argument of periapsis values. Their lifetimes vary from 40165 days, demonstrating that arbitrarily placing a spacecraft in a lowaltitude, near-polar orbit can result in impact after a relatively short period of time. The instability causing these short lifetimes arises primarily from the perturbations on the spacecraft from Jupiter. Therefore, this paper is motivated by the desire to design orbits about Europa that have long lifetimes.

To study orbits about Europa, we use a modified form of the Hill three-body problem with the tidal force from Jupiter and the $J_{2}, C_{22}$, and $J_{3}$ gravity effects of Europa. These particular gravity effects are included because they are the largest effect on the motion in the case of Europa. This system has 3 degrees of freedom and therefore obtaining a qualitative understanding of the motion is very difficult. To simplify the analysis, we remove degrees of freedom by averaging. The first averaging is over the mean anomaly of the orbit of the spacecraft about Europa, and reduces the system to 2 degrees

Received 16 August 2005; revision received 1 January 2006; accepted for publication 2 January 2006. Copyright (C) 2006 by the American Institute of Aeronautics and Astronautics, Inc. All rights reserved. Copies of this paper may be made for personal or internal use, on condition that the copier pay the $\$ 10.00$ per-copy fee to the Copyright Clearance Center, Inc., 222 Rosewood Drive, Danvers, MA 01923; include the code $\$ 10.00$ in correspondence with the CCC

*Ph.D. Candidate; mpaskowi@ umich.edu. Student Member AAS, Student Member AIAA.

†Associate Professor; scheeres@umich.edu. Member AAS, Associate Fellow AIAA. of freedom. The second averaging is over the mean anomaly of the orbit of Europa about Jupiter, and reduces the system to 1 degree of freedom. The 1 degree-of-freedom (DOF) system is then used to understand the underlying dynamics of the full 3-DOF system.

In the 1-DOF system, orbits that have constant orbit elements on average are identified, called frozen orbits. Previous work using averaging methods has been done to study the dynamics of orbits about planetary satellites and frozen orbits. Broucke [2] studied orbit dynamics in a model that included the tidal force. He found elliptic and circular frozen orbits and investigated their stability. Scheeres et al. [1] studied near-circular frozen orbits in a model that included both the tidal force and $J_{2}$. They investigated the stability of these orbits and also developed an analytic theory to postpone impact with the surface for significant periods of time. In addition San-Juan et al. [3] and Lara et al. [4] studied orbit dynamics about planetary satellites using a more rigorous averaging method that involved Lie transforms to develop averaged equations to a higher order. They included the tidal term and $J_{2}$ and the tidal term, $J_{2}$ and $C_{22}$, respectively.

The inclusion of the $J_{3}$ Europa gravity effect in our system causes additional frozen orbits to arise. In particular, frozen orbits with small but nonzero eccentricities and near-polar inclinations exist. These orbits are most likely to satisfy the science orbit requirements for a mission to Europa; however, they are unstable. Because they have nonzero eccentricities, their stable and unstable manifolds are shifted and this causes variations in the rate of change of the eccentricity along their paths. These variations can be exploited by choosing the path that remains at near-circular eccentricities for the longest period of time. If a trajectory in the full 3-DOF system follows this path, it can have a relatively long lifetime on the order of 150 days, as compared with lifetimes as short as 40 days for randomly chosen initial conditions.

After identifying the desired trajectory in the 1-DOF system, we develop a method to systematically compute initial conditions in the 3-DOF system such that the trajectory in that system will follow the 1-DOF system on average. To do this, we use all three systems identified thus far, namely, the 1,2, and 3-DOF systems. We start with a point on the stable manifold of a frozen orbit in the 1-DOF system. We then linearize the 1-DOF system about that point on the manifold and obtain corrections such that the initial conditions lead to a trajectory in the 2-DOF system that has motion in the 1-DOF system as its average. This procedure is repeated on the $3-\mathrm{DOF}$ system, by linearizing about the corrected point in the 2-DOF system. We end up with a set of initial conditions in the 3-DOF system that, 


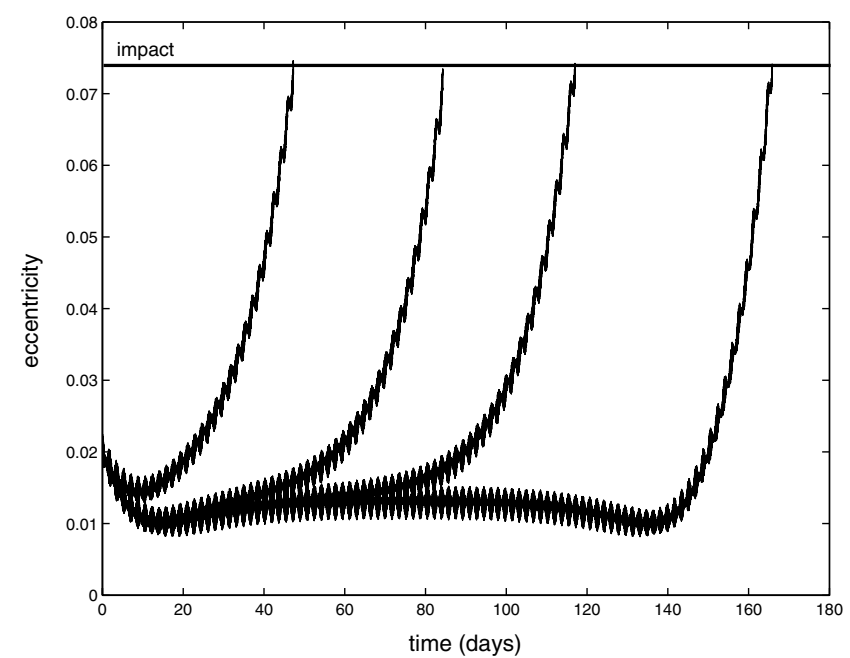

Fig. 1 Eccentricity as a function of time for four trajectories with different lifetimes.

when integrated, follows the 1-DOF system on average. However, it is necessary to introduce a bias in one of the variables to ensure that the correct path in the 1-DOF system is followed. This bias can be computed via a numerical search over a small range of values. Once the bias is included, the set of initial conditions in the 3-DOF system results in a long lifetime orbit.

\section{Definition of the System}

The physical situation we consider consists of a spacecraft in orbit about a planetary satellite which is in turn in orbit about a planet (see Fig. 2). In particular, we consider a spacecraft orbiting Jupiter's moon Europa. We include the first order perturbing effect of the planet's gravity on the planetary satellite, as well as the $J_{2}, C_{22}$, and $J_{3}$ gravity effects of the planetary satellite. We chose to include those three terms from Europa's gravity field because they have been found to strongly influence the dynamics and orbit lifetimes. Note that when including $C_{22}$, we assume Europa's rotation rate about its maximum moment of inertia is synchronous with its orbit rate and that its axis with minimum moment of inertia is aligned with the Jupiter-Europa line. The perturbing potential is the addition of the four contributing perturbations $[5,6]$ :

$$
\begin{aligned}
R & =\frac{1}{2} N^{2}\left(3 x^{2}-r^{2}\right)-\frac{\mu J_{2}}{2}\left(\frac{3 z^{2}}{r^{5}}-\frac{1}{r^{3}}\right)+\frac{3 \mu C_{22}}{r^{5}}\left(x^{2}-y^{2}\right) \\
& -\frac{\mu J_{3}}{2}\left(\frac{5 z^{3}}{r^{7}}-\frac{3 z}{r^{5}}\right)
\end{aligned}
$$

This potential corresponds to a modified form of Hill's three-body problem, incorporating gravity coefficients for the central attracting body. The general equations of motion for a system in this form are

$$
\begin{aligned}
\ddot{x}+2 N \dot{y} & =-\frac{\mu}{r^{3}} x+N^{2} x+\frac{\partial R}{\partial x} \\
\ddot{y}-2 N \dot{x} & =-\frac{\mu}{r^{3}} y+N^{2} y+\frac{\partial R}{\partial y} \\
\ddot{z} & =-\frac{\mu}{r^{3}} z+\frac{\partial R}{\partial z}
\end{aligned}
$$

where $x$ is measured along the line from the planet to the planetary satellite, $r$ is the orbital radius from the center of the planetary satellite, and $N$ and $\mu$ are the orbit angular rate and gravitational parameter, respectively, of the planetary satellite. Then, using the perturbing potential from Eq. (1), we obtain the following equations of motion:

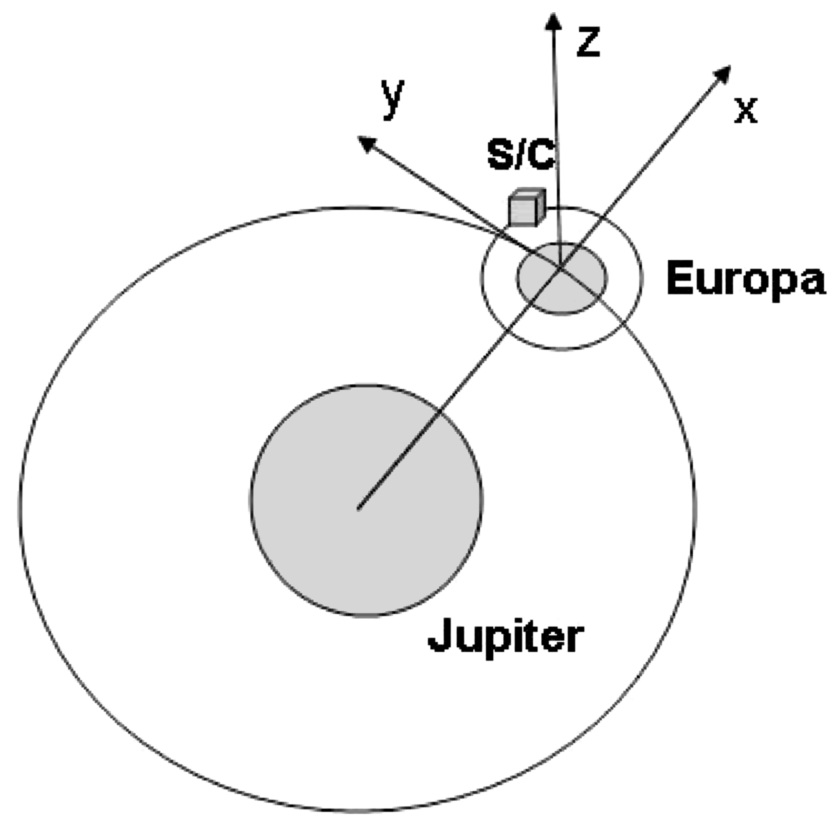

Fig. 2 Geometry of the Europa orbiter model.

$$
\begin{aligned}
& \ddot{x}+2 N \dot{y}=-\frac{\mu}{r^{3}} x+3 N^{2} x-\frac{\mu J_{2} x}{2 r^{5}}\left[3-15\left(\frac{z}{r}\right)^{2}\right] \\
& +\frac{3 \mu C_{22} x}{r^{5}}\left(2-\frac{5\left(x^{2}-y^{2}\right)}{r^{2}}\right)-\frac{\mu J_{3} x z}{2 r^{7}}\left[15-35\left(\frac{z}{r}\right)^{2}\right] \\
& \ddot{y}-2 N \dot{x}=-\frac{\mu}{r^{3}} y-\frac{\mu J_{2} y}{2 r^{5}}\left[3-15\left(\frac{z}{r}\right)^{2}\right] \\
& -\frac{3 \mu C_{22} y}{r^{5}}\left(2+\frac{5\left(x^{2}-y^{2}\right)}{r^{2}}\right)-\frac{\mu J_{3} y z}{2 r^{7}}\left[15-35\left(\frac{z}{r}\right)^{2}\right] \\
& \ddot{z}=-\frac{\mu}{r^{3}} z-N^{2} z-\frac{\mu J_{2} z}{2 r^{5}}\left[9-15\left(\frac{z}{r}\right)^{2}\right] \\
& -\frac{15 \mu C_{22} z}{r^{7}}\left(x^{2}-y^{2}\right)-\frac{\mu J_{3}}{2 r^{5}}\left[-3+30\left(\frac{z}{r}\right)^{2}-35\left(\frac{z}{r}\right)^{4}\right]
\end{aligned}
$$

In the following, we refer to Eqs. (5-7) as defining the 3-DOF system. Solutions in the 3 -DOF system are found by numerically integrating these equations. This system has the Jacobi integral, which in Cartesian coordinates is stated as

$$
J=\frac{1}{2}\left(\dot{x}^{2}+\dot{y}^{2}+\dot{z}^{2}\right)-\frac{\mu}{r}-\frac{1}{2} N^{2}\left(x^{2}+y^{2}\right)-R
$$

\section{Reduction of the System by Averaging}

The general system, governed by the potential of Eq. (1), has 3 degrees of freedom. The system can be characterized in terms of the six inertial orbital elements $(a, e, i, \omega, \Omega, M)$ which are the semimajor axis, eccentricity, inclination, argument of periapsis, longitude of the ascending node, and mean anomaly, respectively. It is also useful to characterize the system in terms of the Delaunay elements, which are defined as

$$
\begin{aligned}
L & =\sqrt{\mu a}, & & M \\
G & =L \sqrt{1-e^{2}}, & & \omega \\
H & =G \cos i, & & \Omega
\end{aligned}
$$

The Delaunay variables are used here because they aid in the discussion of the reduction of the system by averaging that follows. 
The equations of motion in this system are stated as [5]

$$
\begin{array}{llrl}
\frac{\mathrm{d} L}{\mathrm{~d} t}=\frac{\partial R}{\partial M} & \frac{\mathrm{d} M}{\mathrm{~d} t}=n-\frac{\partial R}{\partial L} & \frac{\mathrm{d} G}{\mathrm{~d} t}=\frac{\partial R}{\partial \omega} \\
\frac{\mathrm{d} \omega}{\mathrm{d} t}=-\frac{\partial R}{\partial G} & \frac{\mathrm{d} H}{\mathrm{~d} t}=\frac{\partial R}{\partial \Omega} & \frac{\mathrm{d} \Omega}{\mathrm{d} t}=-\frac{\partial R}{\partial H}
\end{array}
$$

The 3 degrees of freedom refer to the three free angular coordinates, $M, \omega$, and $\Omega$, and $L, G$, and $H$ are their corresponding momenta. In particular, $G$ is the angular momentum and $H$ is the $z$ component of the angular momentum. The Jacobi integral, defined in Eq. (8) can be expressed in osculating orbital elements as

$$
J=-\frac{\mu}{2 a}-N H-R(a, e, i, \omega, \Omega, M)
$$

Averaging is a technique that can be used to remove degrees of freedom from this system. In general, averaging occurs over an angle of the system and causes its corresponding momentum to be conserved. We first reduce the system to 2 degrees of freedom by averaging over the mean anomaly of the spacecraft about Europa:

$$
\bar{R}=\frac{1}{2 \pi} \int_{0}^{2 \pi} R \mathrm{~d} M
$$

The computation of $\bar{R}$ requires the definition of the Cartesian coordinates $x, y$, and $z$ as

$$
\begin{gathered}
x=r[\cos (\omega+v) \cos \tilde{\Omega}-\sin (\omega+v) \sin \tilde{\Omega} \cos i] \\
y=r[\cos (\omega+v) \sin \tilde{\Omega}+\sin (\omega+v) \cos \tilde{\Omega} \cos i] \\
z=r \sin i \sin (\omega+v)
\end{gathered}
$$

where $\tilde{\Omega}=\Omega-N\left(t-t_{0}\right)$ is the longitude of the ascending node relative to the rotating coordinate frame, $t$ is the time, and $t_{0}$ is an initial epoch. The averaged potential is found to be

$$
\begin{aligned}
\bar{R} & =\frac{N^{2} a^{2}}{4}\left\{\left(1-\frac{3}{2} \sin ^{2} i+\frac{3}{2} \cos 2 \tilde{\Omega} \sin ^{2} i\right)\left(1+\frac{3}{2} e^{2}\right)\right. \\
& +\frac{15}{4} e^{2} \cos 2 \omega\left[\sin ^{2} i+\cos 2 \tilde{\Omega}\left(1+\cos ^{2} i\right)\right] \\
& \left.-\frac{15}{2} e^{2} \sin 2 \omega \sin 2 \tilde{\Omega} \cos i\right\}+\frac{\mu J_{2}}{2 a^{3}\left(1-e^{2}\right)^{3 / 2}}\left(1-\frac{3}{2} \sin ^{2} i\right) \\
& +\frac{3 \mu C_{22}}{2 a^{3}\left(1-e^{2}\right)^{3 / 2}} \cos 2 \tilde{\Omega} \sin ^{2} i \\
& +\frac{3 \mu J_{3}}{2 a^{4}\left(1-e^{2}\right)^{5 / 2}} e \sin \omega \sin i\left(1-\frac{5}{4} \sin ^{2} i\right)
\end{aligned}
$$

Because we averaged over the mean anomaly, it is no longer present in the potential defined by Eq. (16). The equation for the timederivative of the semimajor axis in this averaged system is [7]

$$
\frac{\mathrm{d} a}{\mathrm{~d} t}=\frac{2}{n a} \frac{\partial \bar{R}}{\partial M} \equiv 0
$$

Therefore, the semimajor axis is now constant and acts as another integral of motion. We have reduced the system to a 2-DOF system consisting of the pairs $(H, \Omega)$ and $(G, \omega)$.

The 2-DOF system is a time-periodic function of $\tilde{\Omega}$. This makes initial analysis of the qualitative behavior of the system difficult and so we reduce it further by performing another averaging, over the orbit of Europa about Jupiter. Because $\tilde{\Omega}$ is uniformly decreasing with time due to its definition with respect to the rotating coordinate frame, it can be used as an independent variable in place of the time. Therefore, we can average the dynamics again over this new independent variable to find the simplest form of our dynamics:

$$
\tilde{R}=\frac{1}{2 \pi} \int_{0}^{2 \pi} \bar{R} \mathrm{~d} \tilde{\Omega}
$$

This defines the doubly averaged potential:

$$
\begin{aligned}
\tilde{R} & =\frac{N^{2} a^{2}}{4}\left[\left(1-\frac{3}{2} \sin ^{2} i\right)\left(1+\frac{3}{2} e^{2}\right)+\frac{15}{4} e^{2} \cos 2 \omega \sin ^{2} i\right] \\
& +\frac{\mu J_{2}}{2 a^{3}\left(1-e^{2}\right)^{3 / 2}}\left(1-\frac{3}{2} \sin ^{2} i\right) \\
& +\frac{3 \mu J_{3}}{2 a^{4}\left(1-e^{2}\right)^{5 / 2}} e \sin \omega \sin i\left(1-\frac{5}{4} \sin ^{2} i\right)
\end{aligned}
$$

This potential has only tidal, $J_{2}$, and $J_{3}$ components because the $C_{22}$ component vanishes under the averaging. Although this means that the effect of $C_{22}$ is not included during the analysis of this system, we do consider its effect in later sections when we remove the averaging. Because $\Omega$ is now eliminated, an additional integral of motion exists for this system, the $z$-component of the angular momentum defined by Eq. (9). Because $a$ was constant in the 2-DOF system it is also constant in this system, and so we define the simpler functional form of the integral $\theta=H^{2} / \mu a$, or [2]

$$
\theta=\left(1-e^{2}\right) \cos ^{2} i
$$

Therefore, the system governed by the doubly averaged potential in Eq. (19) is a 1-DOF system in $(G, \omega)$. To simplify later analysis, recall the Jacobi integral which, because it was an integral of motion in the full 3-DOF system, is also in integral of motion here and has the same value as before the averaging because it is a constant. Therefore, Eq. (11) implies that

$$
J=-\frac{\mu}{2 a}-N H-\tilde{R}(e, i, \omega)
$$

Because $a$ and $H$ are integrals of motion in this system, we conclude that the potential of the $1-\mathrm{DOF}$ system, $\tilde{R}$, is also an integral of motion for our reduced 1-DOF system. Note that this is not true for the potentials of the 2- and 3-DOF systems.

\section{Frozen Orbits in the 1-DOF System}

We will now perform a detailed study of the 1-DOF system. The equations of motion of these two variables can be obtained from the 1-DOF potential $\tilde{R}$ as follows:

$$
\begin{gathered}
\frac{\mathrm{d} G}{\mathrm{~d} t}=\frac{\partial \tilde{R}}{\partial \omega} \\
\frac{\mathrm{d} \omega}{\mathrm{d} t}=-\frac{\partial \tilde{R}}{\partial G}
\end{gathered}
$$

Because $\tilde{R}$ is also a constant, we note that this system can be formally reduced to quadratures. However, for our analysis, it is more useful to consider the time derivatives of the osculating orbital elements $e, i$, and $\omega$, where we note that the equations for $e$ and $i$ can be combined into the equation for $G$. These equations are obtained by substituting the 1-DOF potential, Eq. (19) into the Lagrange planetary equations [7].

$$
\begin{aligned}
\frac{\mathrm{d} e}{\mathrm{~d} t} & =\frac{15}{8} \frac{N^{2}}{n} e \sqrt{1-e^{2}} \sin ^{2} i \sin 2 \omega \\
& -\frac{3 J_{3} n}{2 a^{3}\left(1-e^{2}\right)^{3}} \sin i\left(1-\frac{5}{4} \sin ^{2} i\right) \cos \omega \\
\frac{\mathrm{d} i}{\mathrm{~d} t} & =-\frac{15}{16} \frac{N^{2}}{n} \frac{e^{2}}{\sqrt{1-e^{2}}} \sin 2 i \sin 2 \omega \\
& +\frac{3 \mu J_{3}}{2 n a^{6}\left(1-e^{2}\right)^{3}} e \cos i\left(1-\frac{5}{4} \sin ^{2} i\right) \cos \omega
\end{aligned}
$$




$$
\begin{aligned}
\frac{\mathrm{d} \omega}{\mathrm{d} t} & =\frac{3}{8} \frac{N^{2}}{n} \frac{1}{\sqrt{1-e^{2}}}\left[5 \cos ^{2} i-1+5 \sin ^{2} i \cos 2 \omega\right. \\
& \left.+e^{2}(1-5 \cos 2 \omega)\right]+\frac{3 n J_{2}}{4 a^{2}\left(1-e^{2}\right)^{2}}\left(1-\frac{5}{4} \sin ^{2} i\right) \\
& +\frac{3 J_{3} n}{2 a^{3}\left(1-e^{2}\right)^{3}} \frac{\sin \omega \sin i}{e}\left[\left(1-\frac{5}{4} \sin ^{2} i\right)\left(1+4 e^{2}\right)\right. \\
& \left.-\frac{e^{2}}{\sin ^{2} i}\left(1-\frac{19}{4} \sin ^{2} i+\frac{15}{4} \sin ^{4} i\right)\right]
\end{aligned}
$$

If we examine Eqs. (24-26), we observe that equilibrium solutions to these equations exist. We denote these equilibria as frozen orbits. These orbits are not frozen in space, but have constant values of $e, i$, and $\omega$ on average. As previously noted, the longitude of the ascending node does not affect any of the other orbital elements and it cannot be made frozen, so we need not consider it when evaluating frozen orbits.

The equilibrium solutions need to be determined numerically due to the complexity of the equations. Therefore, because they will be functions of the semimajor axis, we must choose a numerical semimajor axis value. However, because we desire an orbit with a low periapsis, we instead choose a numerical value for the radius of periapsis, $r_{p}$, and use the following relation to eliminate the semimajor axis from the equations:

$$
a=\frac{r_{p}}{1-e}
$$

If we first consider Eqs. (24) and (25), we see that they are in equilibrium at $\omega= \pm \pi / 2$. Finding the frozen orbit solutions then reduces to solving Eq. (26) with $\omega= \pm \pi / 2$. However, when we examine Eq. (26) we see that its $J_{3}$ component contains a " $\sin \omega$ " term and so there is no symmetry between positive and negative values of $\omega$. Therefore, when solving for the frozen orbit solutions, we must consider $\omega=\pi / 2$ and $\omega=-\pi / 2$ separately. However, there does exist a symmetry between the sign of $J_{3}$ and $\omega$, where changing the sign of $J_{3}$ is equivalent to shifting $\omega$ by $\pi$. By finding the frozen orbit solutions for both frozen values of $\omega$ we allow the analysis to be valid for any value of $J_{3}$. Because at this time accurate values of $J_{3}$ for Europa are not available, we perform our analysis using a particular value of $J_{3}$ that is positive (see Table 1). Note that the Europa gravity coefficients in Table 1 are nondimensional but not normalized. The equation that we solve to find when $\dot{\omega}=0$ is

$$
\begin{aligned}
0= & \frac{3}{8} \frac{N^{2}}{n} \frac{1}{\sqrt{1-e^{2}}}\left(4-10 \sin ^{2} i+6 e^{2}\right)+\frac{3 n J_{2}}{4 a^{2}\left(1-e^{2}\right)^{2}} \\
& \times\left(1-\frac{5}{4} \sin ^{2} i\right) \pm \frac{3 J_{3} n}{2 a^{3}\left(1-e^{2}\right)^{3}} \frac{\sin i}{e} \\
& \times\left[\left(1-\frac{5}{4} \sin ^{2} i\right)\left(1+4 e^{2}\right)\right. \\
& \left.-\frac{e^{2}}{\sin ^{2} i}\left(1-\frac{19}{4} \sin ^{2} i+\frac{15}{4} \sin ^{4} i\right)\right]
\end{aligned}
$$

where the \pm on the $J_{3}$ term corresponds to $\omega= \pm \pi / 2$. This equation can be solved by using the $\theta$ integral of motion and eliminating either

Table 1 Parameters of Europa

\begin{tabular}{lcc}
\hline \hline \multicolumn{1}{c}{ Parameter } & Symbol & Value \\
\hline Europa radius & $R_{E}$ & $1560.8 \mathrm{~km}$ \\
Europa orbital period & $T$ & $355 \mathrm{days}$ \\
Europa orbit rate & $N$ & $2.05 \times 10^{-5} \mathrm{rad} / \mathrm{s}$ \\
Europa gravitational parameter & $\mu$ & $3.201 \times 10^{3} \mathrm{~km}^{3} / \mathrm{s}^{2}$ \\
Nondimensional Europa $J_{2}{ }^{\mathrm{a}}$ & $J_{2}$ & $4.2749 \times 10^{-4}$ \\
Nondimensional Europa $C_{22}{ }^{\mathrm{a}}$ & $C_{22}$ & $1.2847 \times 10^{-4}$ \\
Nondimensional Europa $J_{3}{ }^{\mathrm{a}}$ & $J_{3}$ & $1.3784 \times 10^{-4}$ \\
\hline \hline
\end{tabular}

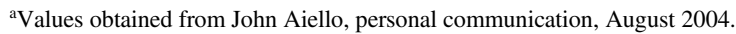

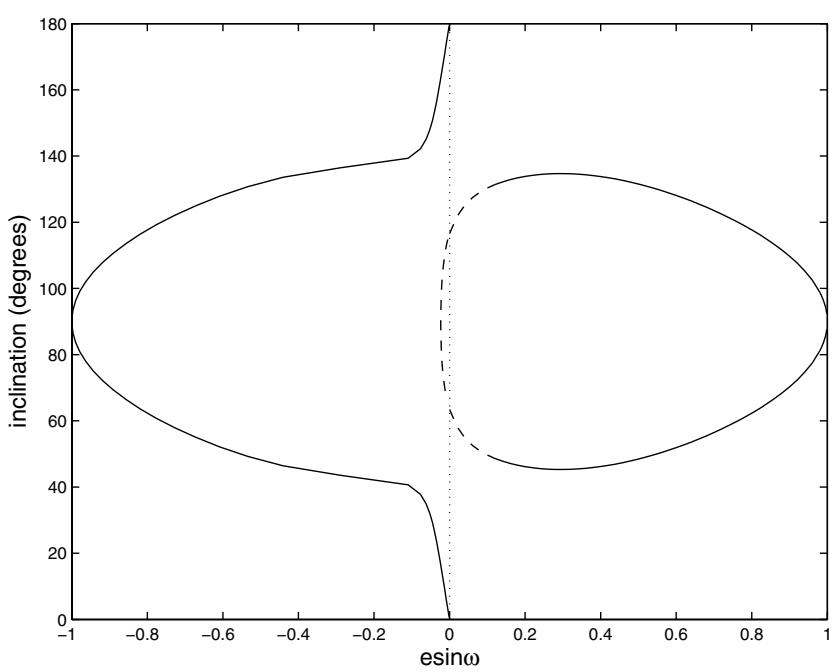

Fig. 3 Stable (solid curves) and unstable (dashed curve) frozen orbit solutions.

$e$ or $i$ from Eq. (28). However, because the range of $\theta$ is not immediately apparent, it is easier to choose values of either $e$ or $i$ and solve Eq. (28) for the other orbital element. The value of $\theta$ can then be obtained from the solution.

Figure 3 shows the frozen orbit solutions for $J_{3}>0$ and $r_{p}=1661 \mathrm{~km}$, which corresponds to a periapsis altitude of $100 \mathrm{~km}$. Note that near-equatorial frozen orbits also exist over all eccentricities for $J_{3} \sin \omega>0(\omega= \pm \pi / 2)$, but these solutions are not included in Fig. 3 because they are not relevant to our discussion. In general, there is no symmetry between the solutions for $\omega= \pm \pi / 2$. However, as the eccentricity increases the frozen orbit solutions for both values of $\omega$ converge to the same solution. This occurs because as the eccentricity increases, the apoapsis of the orbit gets larger and the influences of $J_{2}$ and $J_{3}$ decrease. Previous studies of frozen orbits that include only the tidal force show symmetry in the frozen orbit solutions for $\omega= \pm \pi / 2$ [2]. Therefore, we conclude that the high-eccentricity frozen orbits are not significantly changed by adding $J_{2}$ and $J_{3}$. We also observe that circular frozen orbits exist only at four isolated points, which in this case have inclinations of $0 \mathrm{deg}, 180 \mathrm{deg}, 63.43 \mathrm{deg}$, and $116.56 \mathrm{deg}$, whereas in the tide-only case, circular frozen orbits existed over all inclinations [1]. Note that the frozen orbit inclinations of $63.43 \mathrm{deg}$ and $116.56 \mathrm{deg}$ are close to but not exactly equal to $i=\sin ^{-1}(\sqrt{4 / 5})$ due to the existence of the tidal term. In the case where only $J_{2}$ and $J_{3}$ are included, these circular frozen orbits exist independently of the parameter values [1]. However, including the tidal term as we do here breaks this relationship and the frozen orbits with $i=\sin ^{-1}(\sqrt{4 / 5})$ have nonzero eccentricities. The breaking of this relationship also causes the circular frozen orbits to have inclinations slightly different from $i=\sin ^{-1}(\sqrt{4 / 5})$ that depend on the values of $N$ and $\mu$ and the existence of the $J_{2}$ and $J_{3}$ components. Other types of frozen orbits that exist are near-circular, near-polar and near-circular, nearequatorial for $\omega=-90 \mathrm{deg}$ when $J_{3}>0$. Finally, midinclination ( 20-50 deg and $\sim 130-160 \mathrm{deg}$ ) circular or near-circular frozen orbits do not exist when the effects of both $J_{2}$ and $J_{3}$ are considered.

\section{Stability of Frozen Orbit Solutions}

To determine the stability of the frozen orbit solutions, we linearize Eqs. (24-26) about the frozen orbit solutions. Define $i=i^{*}+\delta i, e=e^{*}+\delta e$, and $\omega=\omega^{*}+\delta \omega$ where $e^{*}, i^{*}$, and $\omega^{*}$ are the frozen orbit values of these orbital elements. The resulting equations are very complicated, and so we represent them by

$$
\begin{aligned}
& \dot{\delta i}=f\left(e^{*}, i^{*}, \omega^{*}\right) \delta \omega \\
& \dot{\delta} e=g\left(e^{*}, i^{*}, \omega^{*}\right) \delta \omega
\end{aligned}
$$

$$
\dot{\delta \omega}=h_{1}\left(e^{*}, i^{*}, \omega^{*}\right) \delta e+h_{2}\left(e^{*}, i^{*}, \omega^{*}\right) \delta i
$$



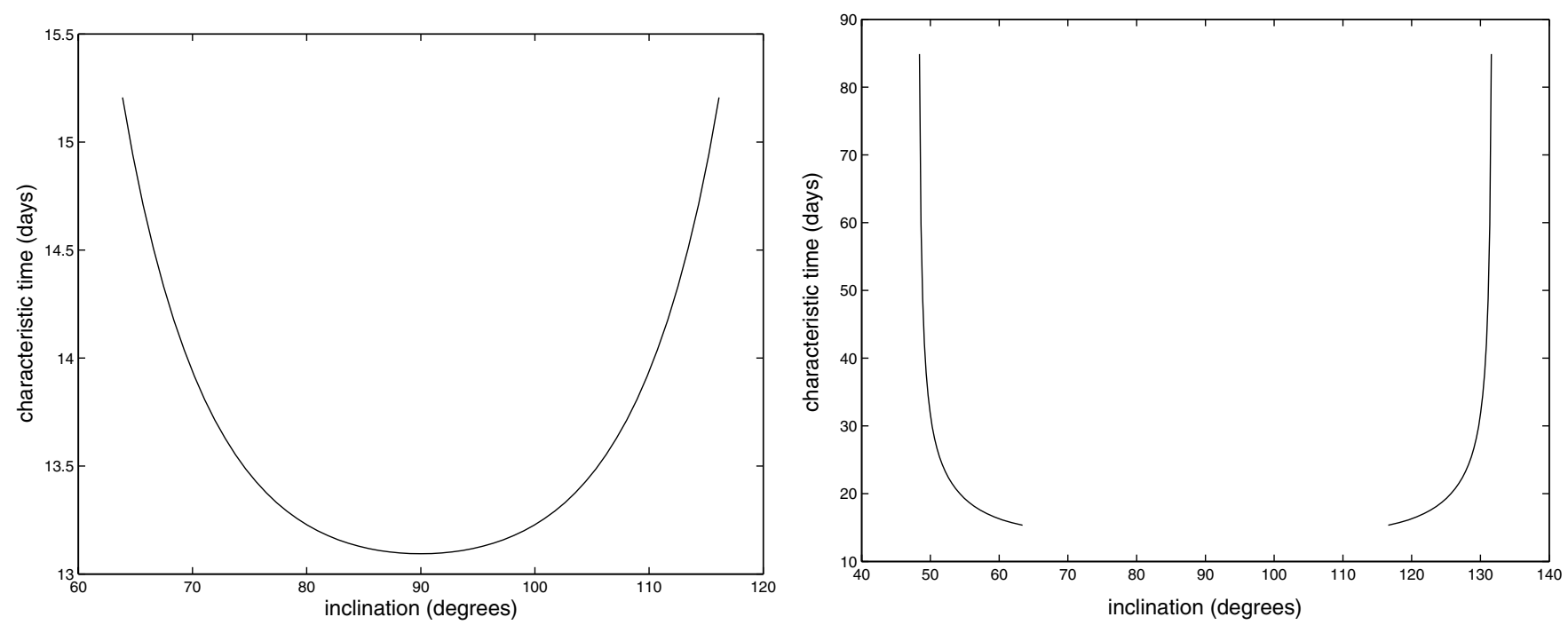

Fig. 4 Characteristic time for near-circular, near-polar (left) and elliptic midinclination (right) unstable frozen orbits.

Because the motions of both $i$ and $e$ are functions of $\omega$ only, the second time derivative of $\delta \omega$ yields a simple second order linear differential equation that has the form of a simple harmonic oscillator:

$$
\ddot{\delta \omega}=\Lambda^{2}\left(e^{*}, i^{*}, \omega^{*}\right) \delta \omega
$$

where $\Lambda^{2}\left(e^{*}, i^{*}, \omega^{*}\right)$ is a constant for each frozen orbit. If $\Lambda^{2}\left(e^{*}, i^{*}, \omega^{*}\right)$ is evaluated for each frozen orbit solution, the stability of that frozen orbit can be determined, where a positive value of $\Lambda^{2}$ denotes an unstable frozen orbit and a negative value of $\Lambda^{2}$ denotes a stable frozen orbit. Figure 3, which shows the frozen orbit solutions, also indicates the stability of the orbit. Stable frozen orbits are denoted by a solid line and unstable frozen orbits are denoted by a dashed line.

Additionally, the strength of the instability of the unstable orbits can be measured by the characteristic time. The characteristic time is defined as $\tau=1 / \Lambda$, and is the time interval it takes the eccentricity to increase by a factor of $\exp (1) \sim 2.718$. In general, the eccentricity will increase by an order of magnitude after $\exp (1) \sim 2.718$ characteristic times. There are two classes of unstable frozen orbits, namely the near-circular, near-polar frozen orbits with $\omega=-90 \mathrm{deg}$ for $J_{3}>0$ and the midinclination frozen orbits with $\omega=90 \mathrm{deg}$ for $J_{3}>0$. Figure 4 shows the characteristic times as functions of the inclination for both classes of unstable frozen orbits. For the nearcircular, near-polar frozen orbits, the characteristic time reaches a minimum when the frozen orbit inclination is polar. This makes sense because for this class of frozen orbits, a maximal frozen orbit inclination corresponds to a maximal frozen orbit eccentricity which means that the perturbations on the orbit are stronger. Similar results are obtained for the other class of frozen orbits where the maximum eccentricity also corresponds to the maximum inclination.

\section{Near-Circular, Near-Polar Orbits}

For the remainder of this paper, we focus our attention on the unstable near-circular, near-polar frozen orbits because they are the most likely to satisfy the requirements for a science mission to a planetary satellite. Because these orbits are near-circular they remain at a low altitude for the entire orbit and because they are near-polar they provide the most visual coverage of a planetary satellite. However, these orbits are unstable. This means that a spacecraft in one of these frozen orbits will impact with the planetary satellite after a relatively short period of time. To prolong the period of time before impact, we perform an in-depth study of stable and unstable manifolds of the frozen orbits to identify orbits in the vicinity of the frozen orbits with significantly longer lifetimes.
Because the underlying dynamics are governed by the 1-DOF system, it is important to have a qualitative understanding of the motion in this regime. We define curves with constant values of the potential and plot them in various orbital element representations. We first recall two of the integrals of motion in the 1-DOF system, $\theta$ and $\tilde{R}$, given by Eqs. (19) and (20). Because these are integrals of motion we can define curves where the motion occurs for specific values of them. These curves will be called contour plots and they define the path that the orbital elements follow on average. Because we are considering the 1-DOF system, if the semimajor axis, eccentricity, and argument of periapsis are known, the inclination can be solved for from the integrals of motion. Therefore, the first case of contour plots that we consider is eccentricity as a function of $\omega$. It is not necessary to plot inclination as a function of $\omega$ because the inclination can be determined easily from the eccentricity plot [8].

Another set of orbital elements that can add some insight to our understanding are the semi-equinoctial elements $(h, k)$, where [6]

$$
h=e \sin \omega \quad k=e \cos \omega
$$

This set of variables is particularly useful for the near-circular orbits that we are considering because all of the motion will be concentrated in a small region of possible $(h, k)$ space. The final set of variables that we consider are the Delaunay variables $(G, \omega)$, defined in Eq. (9).

Figures 5-7 show contour plots in the three different regimes. Note that these figures show the resulting dynamics from our 1-DOF system and do not incorporate higher-order averaging effects. The curves represent constant values of the 1 -DOF potential $\tilde{R}$. Both $\theta$ and $a$ are constant over the entire plot and are computed for the frozen orbit with $e^{*}=0.0129, i^{*}=70 \mathrm{deg}$, and $\omega^{*}=-90 \mathrm{deg}$. The curves show how the orbit elements change in the 1-DOF system. It is clear from these figures that this frozen orbit is unstable. This is most obvious from the right-side plot of Fig. 5 where the eccentricity increases very rapidly while the argument of periapsis stays constant. This means that with any small perturbation from the frozen orbit, the eccentricity will begin to increase. Because the semimajor axis is constant along the contours, the radius of periapsis decreases with increasing eccentricity resulting in impact with Europa's surface. In all three Figs. 5-7, there are also regions where stable equilibrium solutions exist (i.e., $\omega=90 \mathrm{deg}, e \sim 0.65$ ). In these libration regions the argument of periapsis oscillates about the equilibrium point rather than circulating as it does in other regions. However, these regions occur at very high eccentricities. Because the semimajor axis is constant for these plots, the large eccentricity implies a very small radius of periapsis that is actually below the surface of Europa. Therefore, the only physically relevant regions on the contour plots are the regions in the vicinity of the frozen orbits. We find that the 

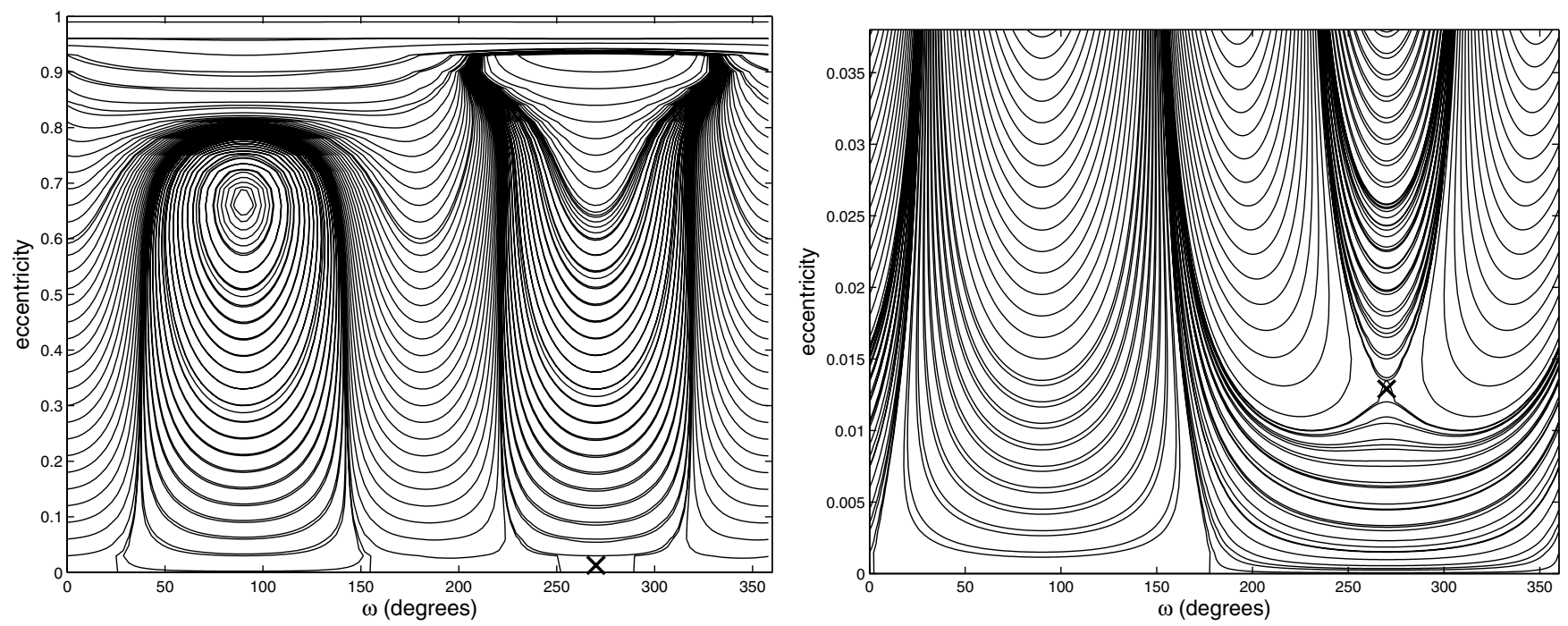

Fig. 5 Contour plot in $(e, \omega)$ space for the frozen orbit identified by "x." Right: Frozen orbit region expansion.

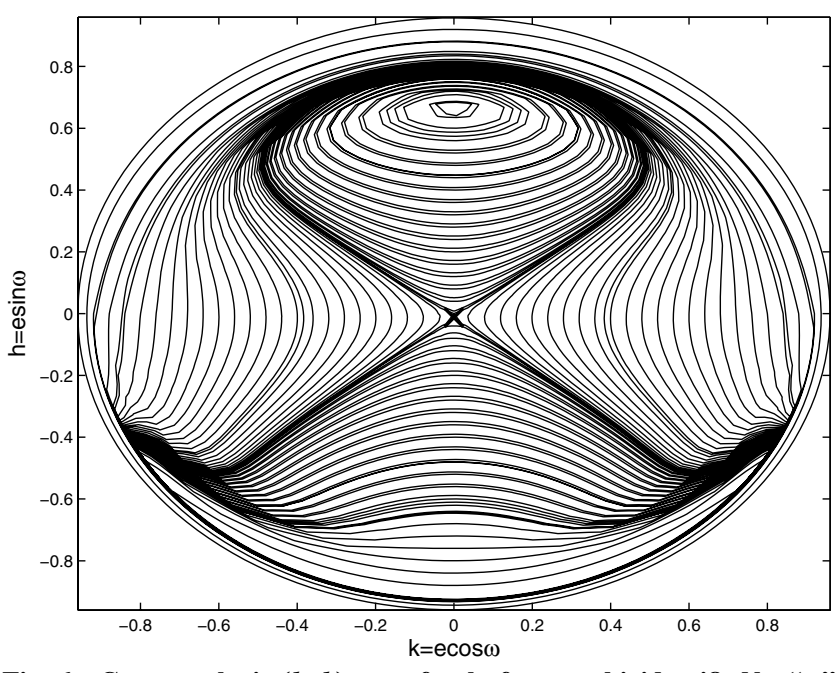

Fig. 6 Contour plot in $(h, k)$ space for the frozen orbit identified by "x."

motion in the full 3-DOF system will follow the curves of the contour plots on average, but not exactly.

Because the 1-DOF system is Hamiltonian and the near-circular, near-polar frozen orbits are unstable, they have both stable and unstable manifolds. The stable manifolds approach the frozen orbit and the unstable manifolds depart from the frozen orbit. If a spacecraft is initialized on the stable manifold of a frozen orbit, we expect that it will drift towards the frozen orbit location on the stable manifold, and then follow the unstable manifold towards impact with the planetary satellite. A good understanding of the manifolds can help us determine where exactly to initialize a spacecraft to obtain the maximum possible lifetime. Note that these manifolds exist only in the 1-DOF case, but their existence here impacts dynamics at higher degrees of freedom.

To compute the manifolds of the frozen orbits, we compute only the contour of constant potential, $\tilde{R}$, that passes through the frozen orbit. This represents the motion in the 1-DOF system corresponding to a trajectory that passes through the frozen orbit. Once again, to have a complete understanding of the situation, we plot the manifolds in the three different regimes, $(h, k),(G, \omega)$, and $(e, \omega)$. Figures 8-10 show the stable and unstable manifolds for a frozen orbit with a $70 \mathrm{deg}$ inclination. In all three figures, the unstable manifolds are numbered I and III and the stable manifolds are numbered II and IV. We can see most clearly from Fig. 8 that there are four possible paths along the manifolds that pass through the frozen orbit, identified with an $\mathrm{x}$. By examining the possible paths along the manifolds in Fig. 9 which is in $(G, \omega)$ space, we can determine how many degrees of $\omega$

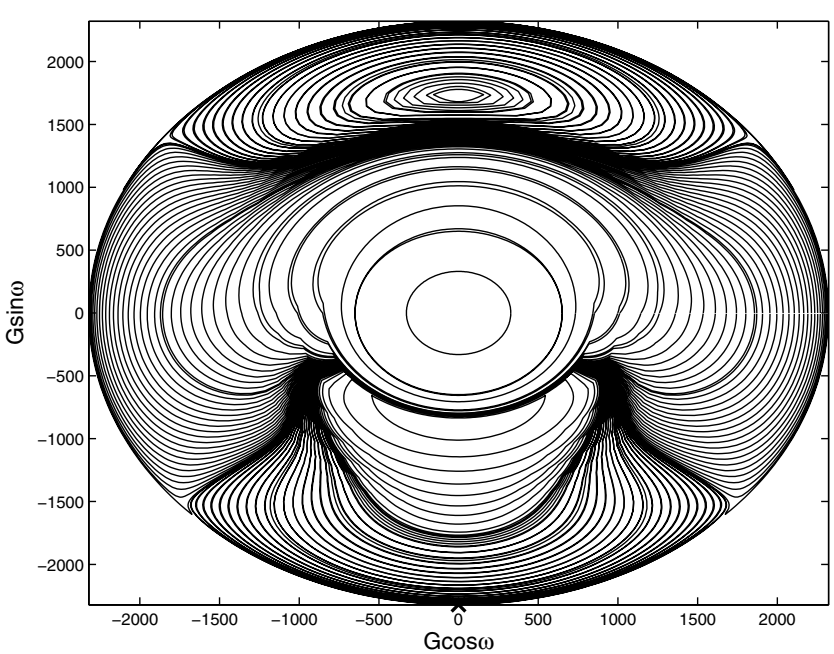

Fig. 7 Contour plot in $(G, \omega)$ space for the frozen orbit identified by "x."

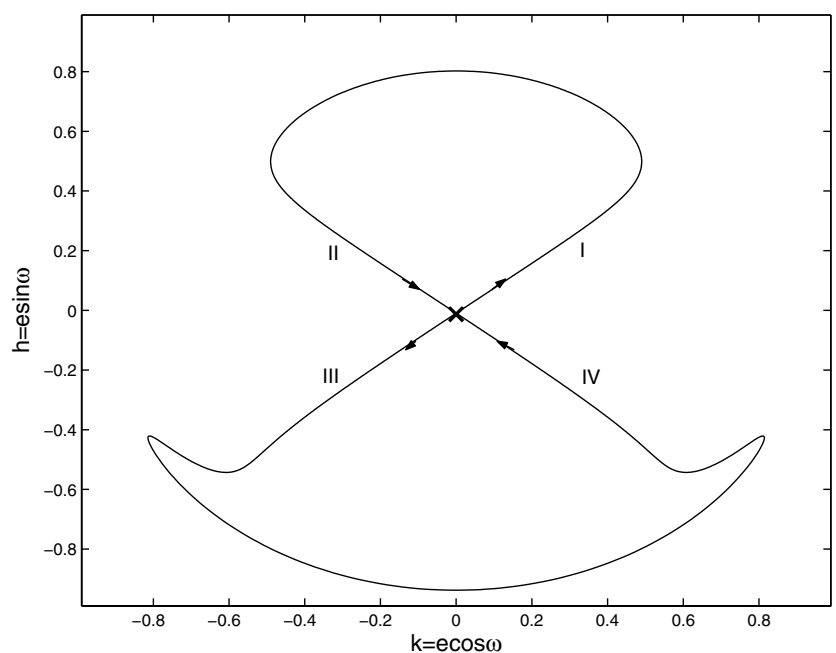

Fig. 8 Stable and unstable manifolds in $(h, k)$ space for the frozen orbit identified by "x."

are covered along each path. For this computation, only the portion of the trajectory that is near-circular is considered. Once the trajectory departs from its near-circular value, the radius of periapsis will decrease to below the surface of the planetary satellite because the 


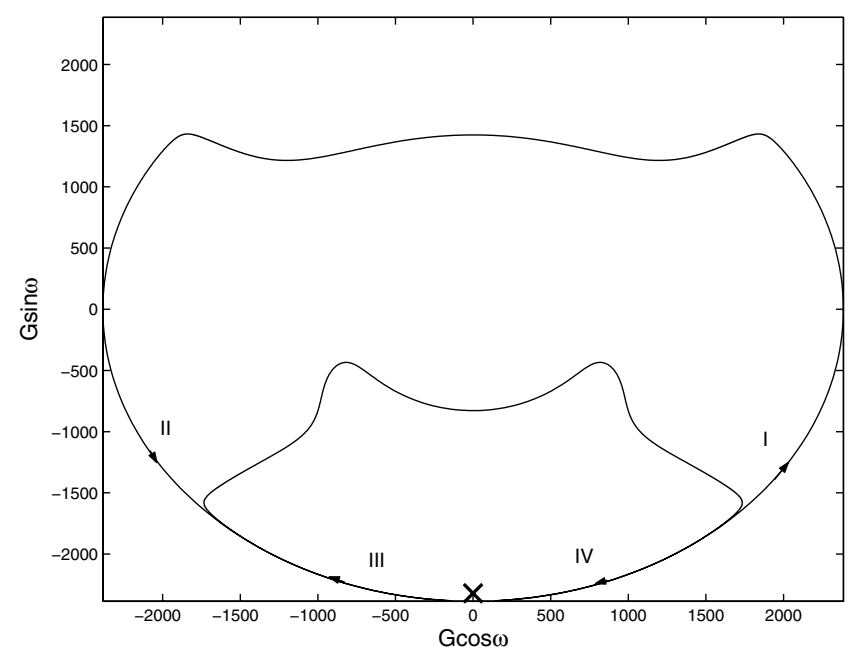

Fig. 9 Stable and unstable manifolds in $(e, \omega)$ space for the frozen orbit identified by "x."

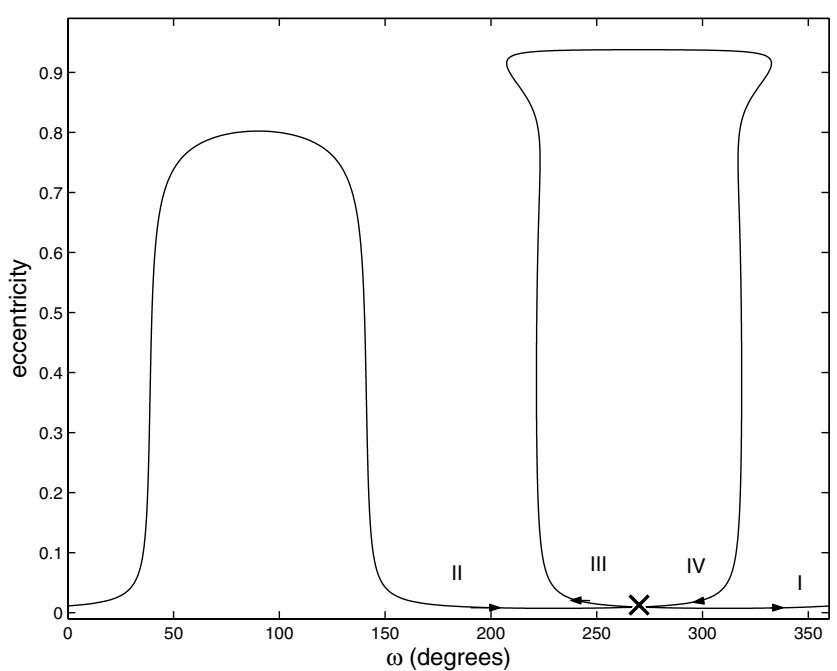

Fig. 10 Stable and unstable manifolds in $(G, \omega)$ space for the frozen orbit identified by "x."

semimajor axis remains constant. The four possible paths and approximate number of degrees of $\omega$ covered along each path are as follows:

1) $\mathrm{II} \rightarrow$ I: $270 \mathrm{deg}$

2 II $\rightarrow$ III: $180 \mathrm{deg}$

3) IV $\rightarrow$ I: $180 \mathrm{deg}$

4) IV $\rightarrow$ III: $90 \mathrm{deg}$

Therefore, by beginning on the stable manifold labeled II, passing through the frozen orbit and then continuing on the unstable manifold labeled I, 270 degrees of $\omega$ are covered while the orbit is near-circular. This is the path that will produce the longest lifetime orbit in the 3-DOF system. Different ranges of $\omega$ are covered along different paths because the frozen orbits are not circular. Figure 11 shows the manifolds in $(h, k)$ space with a circle denoting where the radius of periapsis is at the surface of Europa. Therefore, values of $(h, k)$ outside of the circle represent radii of periapsis below the surface of Europa and hence impact. Different distances along the manifold are covered inside of the circle along the different paths because the frozen orbit located at the center of the $\mathrm{x}$ is not at $(0,0)$. A longer distance inside the circle means a slower rate of change of the eccentricity which leads to a longer lifetime.

In this system, the trajectory will not follow the manifolds exactly; however, we will show that it is possible to determine initial conditions such that this path along the manifold is followed on average and very long lifetime orbits are attained. These manifolds

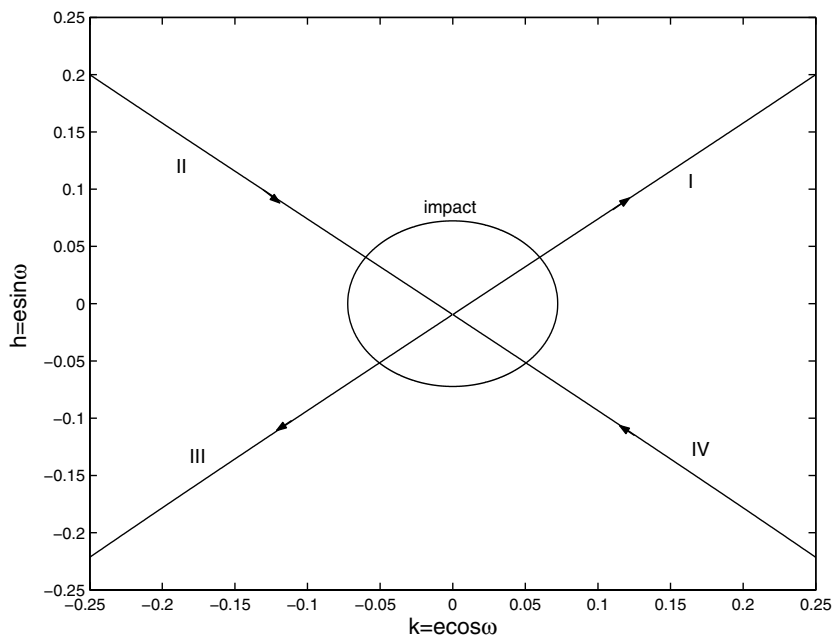

Fig. 11 Stable and unstable manifolds for a frozen orbit and the impact circle.

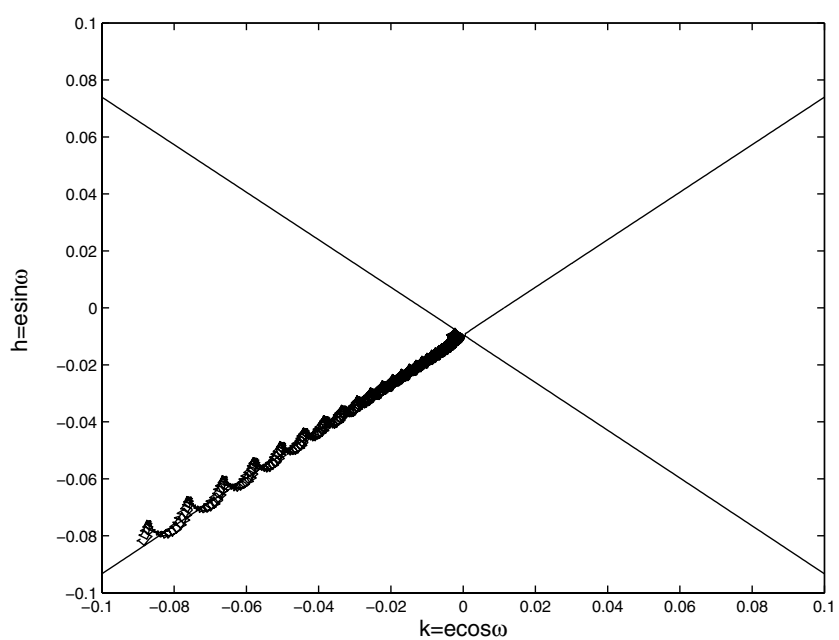

Fig. 12 Integration in 3-DOF system of a trajectory initialized at a frozen orbit.

also explain why when a trajectory with initial conditions corresponding to a frozen orbit is integrated in the unaveraged system, it does not have a particularly long lifetime. As Fig. 12 shows, when the trajectory that is initialized at the frozen orbit location is integrated, it follows the unstable manifold path III because the equilibrium does not exist in the 3-DOF system. Additional lifetime is not gained because the trajectory is not initialized on the stable manifold. This trajectory has a lifetime of 44 days.

\section{Identifying Long Lifetime Orbits}

To this point, we have discussed reducing the 3-DOF system to a 1-DOF system to have a better qualitative understanding of the overall dynamics. From this system, equilibrium solutions are identified and motion in their vicinity is studied. However, as we saw in the preceding section, using the frozen orbits as initial conditions in the full 3-DOF unaveraged system does not produce very long lifetime trajectories.

We have already shown that the longest lifetime orbits will begin on the stable manifold of the frozen orbits, move towards the frozen orbits, and then move off on the unstable manifold. The question then becomes how to determine initial conditions in the 3-DOF system such that the trajectory will follow the manifolds in the 1-DOF system. The easiest first guess would be to initialize the trajectory with initial conditions that lie exactly on the 1-DOF manifold. 
Computing the point on the manifold is fairly straightforward. First a frozen orbit is chosen and the integrals of motions in the 1DOF system, $\theta=\cos ^{2} i^{*}\left(1-e^{* 2}\right)$ and $\tilde{R}^{*}=\tilde{R}\left(e^{*}, i^{*}, \omega^{*}\right)$ are computed. To compute a point on the manifold, we start with a desired initial eccentricity $e_{m}$. Then, having already computed $\theta$ from the frozen orbit conditions, the inclination on the manifold, $i_{m}$ is obtained from

$$
i_{m}=\cos ^{-1} \sqrt{\frac{\theta}{1-e_{m}^{2}}}
$$

Then, substituting $e_{m}$ and $i_{m}$ into

$$
\tilde{R}^{*}=\tilde{R}\left(e_{m}, i_{m}, \omega_{m}\right)
$$

we obtain the following quadratic equation in $\sin \omega_{m}$ :

$$
\begin{aligned}
0= & -\frac{15 N^{2} a^{2}}{8} e_{m}^{2} \sin ^{2} i_{m} \sin ^{2} \omega_{m} \\
& +\frac{3 \mu J_{3}}{2 a^{4}\left(1-e_{m}^{2}\right)^{5 / 2}} e_{m} \sin i_{m}\left(1-\frac{5}{4} \sin ^{2} i_{m}\right) \sin \omega_{m} \\
& +\frac{N^{2} a^{2}}{4}\left[\left(1-\frac{3}{2} \sin ^{2} i_{m}\right)\left(1+\frac{3}{2} e_{m}^{2}\right)+\frac{15}{4} e_{m}^{2} \sin ^{2} i_{m}\right]-\tilde{R}^{*}
\end{aligned}
$$

which can be easily solved for $\omega_{m}$. Continuing with the frozen orbit considered thus far with $e^{*}=0.0129, i^{*}=70 \mathrm{deg}$, and $\omega^{*}=270 \mathrm{deg}$, we compute a point on the 1-DOF manifold with the following characteristics: $e_{m}=0.02, i_{m}=69.998 \mathrm{deg}$, and $\omega_{m}=170.97 \mathrm{deg}$.

As Fig. 13 shows, although the trajectory integrated in the 3-DOF system does follow the manifolds, it is not centered on them and its lifetime is 87 days. This is because there are oscillations due to both the motion of Europa about Jupiter and the spacecraft about Europa that are not accounted for in the 1-DOF system. As well, the effect of $C_{22}$ is not accounted for in the 1-DOF system because averaging cancels it. The ideal trajectory would be centered on the manifold because such a trajectory would spend the longest time in the vicinity of the frozen orbit, giving it the longest possible lifetime. Therefore, we now discuss a method that starts with a point in the 1-DOF system, pulls the point back to a point in the 2-DOF system, and concludes by pulling back that point to initial conditions to be used in the 3-DOF system. These initial conditions take into account all of the oscillations and produce a trajectory that follows the manifolds in the 1-DOF system very closely.

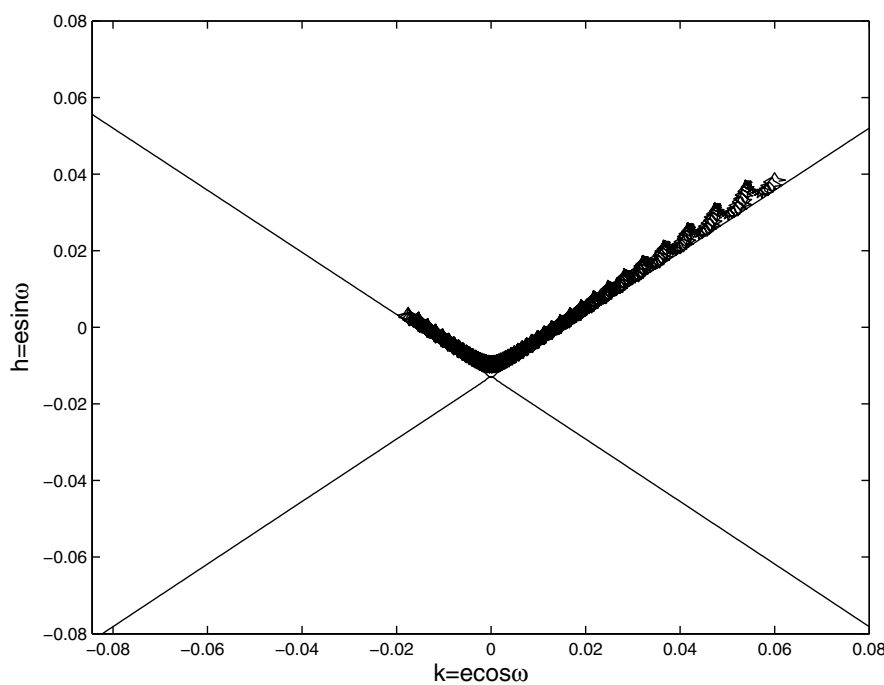

The method that we will use to relate both the 2-DOF system to the 1 -DOF system and the 3-DOF system to the 2-DOF system is a linearization method. We perform it two times, first on the 2-DOF system and then on the 3-DOF system. The method will first be described in general terms, and then some specifics for each system will be discussed.

Let $\mathbf{x}$ be a vector containing the orbital elements under consideration. Then, the time derivatives of the orbital elements, obtained from the Lagrange planetary equations can be expressed as follows:

$$
\mathbf{x}^{\prime}=\mathbf{g}_{0}(\mathbf{x})+\mathbf{g}_{1}(\mathbf{x}, t)
$$

where' denotes the time derivative, $\mathbf{g}_{0}(\mathbf{x})$ is a vector containing secular (averaged) components of the equations, which are timeinvariant, and $\mathbf{g}_{1}(\mathbf{x}, t)$ is a vector containing the time-periodic components of the equations, with period $T$. We assume that $\left|\mathbf{g}_{1}\right| \ll\left|\mathbf{g}_{0}\right|$. We linearize Eq. (36) about a point $\mathbf{x}_{0}$ in the timeinvariant system such that $\mathbf{x}_{0}^{\prime}=\mathbf{g}_{0}\left(\mathbf{x}_{0}\right)$. Then, let $\mathbf{x}=\mathbf{x}_{0}+\delta \mathbf{x}$ where $\delta \mathbf{x}$ is a small perturbation from the point. Substituting this into Eq. (36), we obtain

$$
\left(\mathbf{x}_{0}+\delta \mathbf{x}\right)^{\prime}=\mathbf{g}_{0}\left(\mathbf{x}_{0}+\delta \mathbf{x}\right)+\mathbf{g}_{1}\left(\mathbf{x}_{0}+\delta \mathbf{x}, t\right)
$$

We perform a Taylor series expansion on the right-hand side of Eq. (37), to first order in the time-invariant terms and to zeroth order in the time-periodic terms and obtain

$$
\mathbf{x}_{0}^{\prime}+\delta \mathbf{x}^{\prime}=\mathbf{g}_{0}\left(\mathbf{x}_{0}\right)+\left.\frac{\partial \mathbf{g}_{0}\left(\mathbf{x}_{0}\right)}{\partial \mathbf{x}}\right|_{\mathbf{x}_{0}} \delta \mathbf{x}+\mathbf{g}_{1}\left(\mathbf{x}_{0}, t\right)+\cdots
$$

We carry the expansion to only the zeroth order in the time-periodic terms because the first order terms are much smaller than the zeroth order terms. Doing this allows for a straightforward analysis of the system. The linearized system can be reduced to the following because $\mathbf{x}_{0}^{\prime}=\mathbf{g}_{0}\left(\mathbf{x}_{0}\right)$ :

$$
\delta \mathbf{x}^{\prime}=\mathbf{A} \delta \mathbf{x}+\mathbf{B}(t)
$$

where higher-order terms are ignored and

$$
\begin{aligned}
& \mathbf{A}=\left.\frac{\partial \mathbf{g}_{0}\left(\mathbf{x}_{0}\right)}{\partial \mathbf{x}}\right|_{\mathbf{x}_{0}} \\
& \mathbf{B}(t)=\mathbf{g}_{1}\left(\mathbf{x}_{0}, t\right)
\end{aligned}
$$

Note that we ignore the motion of $\mathbf{x}_{0}$ because we are only interested in evaluating the system over a short time span. Therefore, $\mathbf{A}$ is a

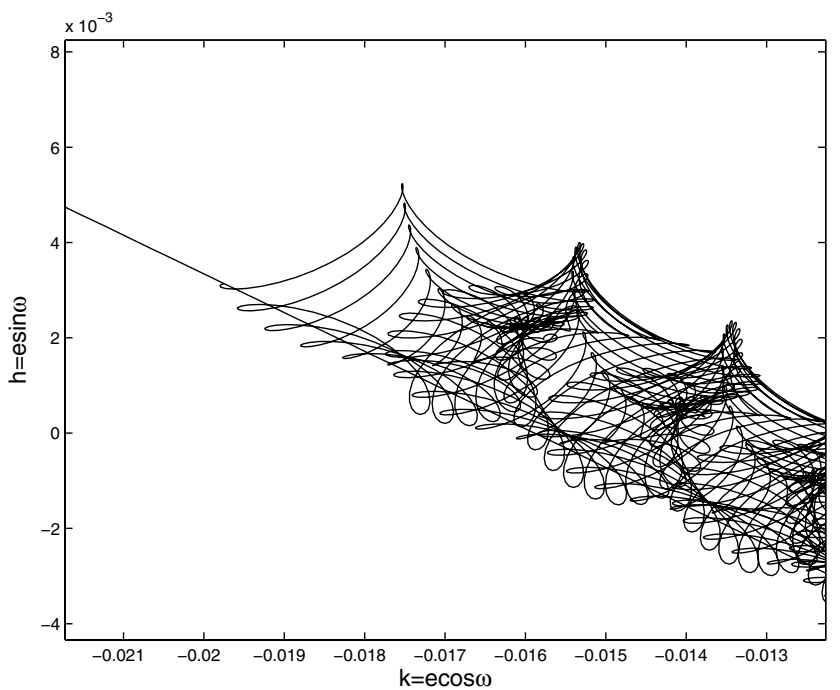

Fig. 13 Left: Integrated trajectory in the 3-DOF system, initialized on the stable manifold of the 1-DOF system. Right: Expansion of the initial part of the trajectory. 
constant matrix and $\mathbf{B}(t)$ is a time-periodic vector. We can solve for $\delta \mathbf{x}$ as follows:

$$
\delta \mathbf{x}=e^{\mathbf{A} t} \delta \mathbf{x}_{0}+\int_{0}^{t} e^{\mathbf{A}(t-\tau)} \mathbf{B}(\tau) \mathrm{d} \tau
$$

Our goal for the linearization is to obtain initial conditions $\delta \mathbf{x}_{0}$ such that the motion in the original system has the motion in the timeinvariant system as its average. To do this, we take the average of Eq. (42) over one period $T$ and set it equal to zero.

$$
0=\frac{1}{T} \int_{0}^{T} e^{\mathbf{A} t} \mathrm{~d} t \delta \mathbf{x}_{0}+\frac{1}{T} \int_{0}^{T} \int_{0}^{t} e^{\mathbf{A}(t-\tau)} \mathbf{B}(\tau) \mathrm{d} \tau \mathrm{d} t
$$

Let

$$
\begin{gathered}
\mathbf{D}=\frac{1}{T} \int_{0}^{T} e^{\mathbf{A} t} \mathrm{~d} t \\
\mathbf{E}=\frac{1}{T} \int_{0}^{T} \int_{0}^{t} e^{\mathbf{A}(t-\tau)} \mathbf{B}(\tau) \mathrm{d} \tau \mathrm{d} t
\end{gathered}
$$

Then, we solve for $\delta \mathbf{x}_{0}$ from Eq. (43) to obtain

$$
\delta \mathbf{x}_{0}=-\mathbf{D}^{-1} \mathbf{E}
$$

Therefore, if we integrate the original system with initial conditions $\mathbf{x}_{0}+\delta \mathbf{x}_{0}$, the motion should be, on average, the time-invariant system initialized at $\mathbf{x}_{0}$.

\section{From 1-DOF System to 2-DOF System}

In this section we apply our algorithm to obtain initial conditions $\delta \mathbf{x}_{0}$ to use in the 2-DOF system that will produce motion that follows the 1-DOF system on average. Because the semimajor axis is constant, we only need to consider

$$
\mathbf{x}=\left[\begin{array}{lll}
e & i & \omega
\end{array}\right]^{T}
$$

The time derivatives of the orbital elements in the 2-DOF system are obtained by applying the Lagrange planetary equations (LPE) to $\bar{R}$ and are as follows [7]:

$$
\begin{gathered}
\frac{\mathrm{d} e}{\mathrm{~d} t}=\frac{1-e^{2}}{n a^{2} e} \frac{\partial \bar{R}}{\partial M}-\frac{\sqrt{1-e^{2}}}{n a^{2} e} \frac{\partial \bar{R}}{\partial \omega} \\
\frac{\mathrm{d} i}{\mathrm{~d} t}=\frac{1}{\sqrt{1-e^{2}} n a^{2}}\left(\cot i \frac{\partial \bar{R}}{\partial \omega}-\csc i \frac{\partial \bar{R}}{\partial \Omega}\right) \\
\frac{\mathrm{d} \omega}{\mathrm{d} t}=\frac{\sqrt{1-e^{2}}}{n a^{2} e} \frac{\partial \bar{R}}{\partial e}-\frac{\cot i}{\sqrt{1-e^{2}} n a^{2} e} \frac{\partial \bar{R}}{\partial i}
\end{gathered}
$$

These equations can be broken up into two components where the $\mathbf{g}_{0}$ components correspond to the LPE applied to $\tilde{R}$ and the $\mathbf{g}_{1}$ components correspond to the LPE applied to $\bar{R}-\tilde{R}$. Then, the $\mathbf{g}_{0}$ components are time-invariant and the $\mathbf{g}_{1}$ components are timeperiodic. The time periodicity appears as periodicity in $\Omega$ with a period of $\pi$. Therefore, to simplify the system, we rewrite Eqs. (4749 ) as derivatives with respect to $\tilde{\Omega}$ rather than derivatives with respect to time by multiplying the right-hand sides of the equations by $-(1 / N)$.

Because our goal is to obtain long lifetime orbits, we linearize the 2-DOF system about a point on the stable manifold of a frozen orbit in the 1-DOF system. Let this point be

$$
\mathbf{x}_{0}^{m}=\left[\begin{array}{lll}
e_{0} & i_{0} & \omega_{0}
\end{array}\right]^{T}
$$

Then, $\mathbf{A}$ and $\mathbf{B}(\tilde{\Omega})$ as defined in Eqs. (40) and (41) can be computed analytically fairly easily using standard built-in Mathematica $\bigcirc$
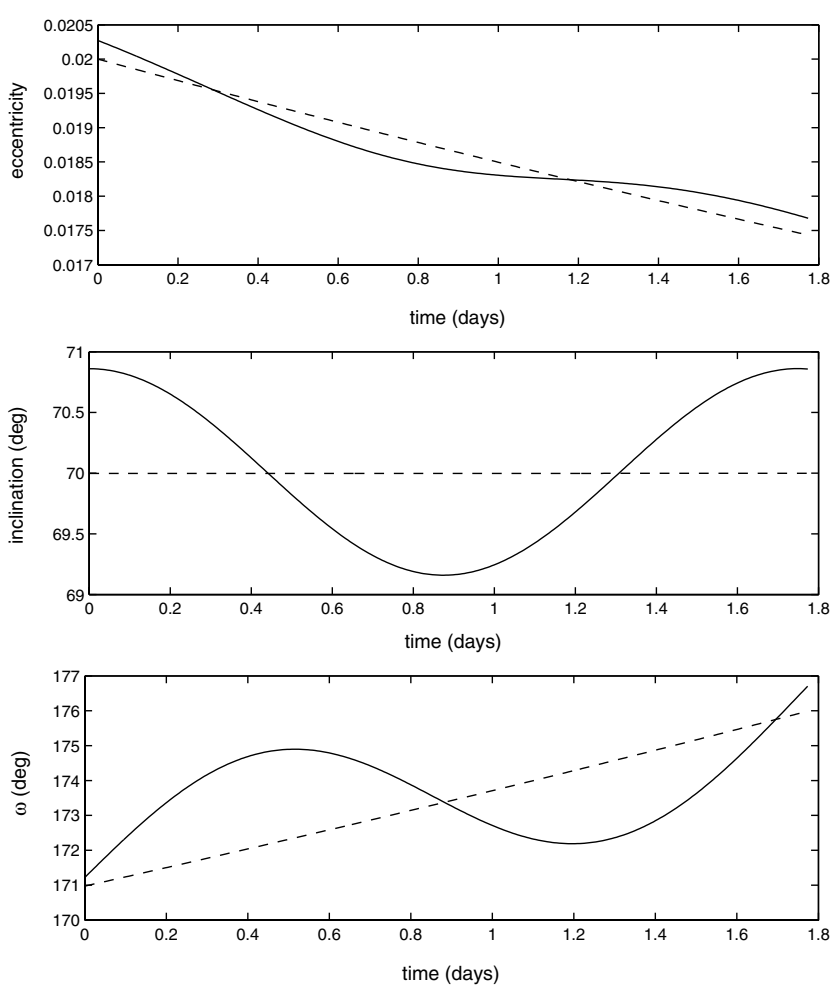

Fig. 14 Time histories of $e, i$, and $\omega$. Solid curves: integrated 2-DOF trajectory. Dashed curves: integrated 1-DOF trajectory.

functions. Following that, $\mathbf{D}$ and $\mathbf{E}$ can be computed to determine the corrections to $\mathbf{x}_{0}^{m}$ and $\delta \mathbf{x}_{0}^{m}$.

We illustrate this method with an example. We first compute the frozen orbit that has $i^{*}=70 \mathrm{deg}, e^{*}=0.0129$, and $\omega^{*}=270 \mathrm{deg}$. We choose the point on the stable manifold of this frozen orbit with the following characteristics:

$$
\mathbf{x}_{0}^{m}=\left[\begin{array}{c}
0.02 \\
69.99756766 \mathrm{deg} \\
170.97339256 \mathrm{deg}
\end{array}\right]
$$

Then, by following the linearization algorithm, we obtain the following corrections to the initial conditions:

$$
\delta \mathbf{x}_{0}^{m}=\left[\begin{array}{c}
0.00027136 \\
0.86310567 \mathrm{deg} \\
0.21413018 \mathrm{deg}
\end{array}\right]
$$

To verify that the 2-DOF trajectory, initialized at $\mathbf{x}_{0}^{m}+\delta \mathbf{x}_{0}^{m}$, has as its average the 1-DOF trajectory initialized at $\mathbf{x}_{0}^{m}$, we plot both the 2DOF and 1-DOF trajectories. As Fig. 14 shows, the dashed lines representing the 1-DOF trajectory pass through the center of the solid curves which represent the 2-DOF trajectory.

\section{From 2-DOF System to 3-DOF System}

In this section, we discuss how to obtain initial conditions to use in the 3-DOF system that will produce motion that follows the 2-DOF system on average. Because we previously found initial conditions to use in the 2-DOF system that produce motion that follows the 1-DOF system on average, the ultimate result of finding initial conditions in the 3-DOF system is that the motion in the 3-DOF system will follow the 1-DOF system on average and the initial conditions we compute will result in orbits that follow the manifold. In this case, there are five equations that must be considered, so

$$
\mathbf{x}=\left[\begin{array}{lllll}
a & e & i & \omega & \Omega
\end{array}\right]^{T}
$$


The time derivatives of these orbital elements, obtained by applying the LPE to $R$ are [7]

$$
\begin{gathered}
\frac{\mathrm{d} a}{\mathrm{~d} t}=\frac{2}{n a} \frac{\partial R}{\partial M} \\
\frac{\mathrm{d} e}{\mathrm{~d} t}=\frac{1-e^{2}}{n a^{2} e} \frac{\partial R}{\partial M}-\frac{\sqrt{1-e^{2}}}{n a^{2} e} \frac{\partial R}{\partial \omega} \\
\frac{\mathrm{d} i}{\mathrm{~d} t}=\frac{1}{\sqrt{1-e^{2}} n a^{2}}\left(\cot i \frac{\partial R}{\partial \omega}-\csc i \frac{\partial R}{\partial \Omega}\right) \\
\frac{\mathrm{d} \omega}{\mathrm{d} t}=\frac{\sqrt{1-e^{2}}}{n a^{2} e} \frac{\partial R}{\partial e}-\frac{\cot i}{\sqrt{1-e^{2}} n a^{2} e} \frac{\partial R}{\partial i} \\
\frac{\mathrm{d} \Omega}{\mathrm{d} t}=\frac{1}{\sqrt{1-e^{2}} n a^{2} \sin i} \frac{\partial R}{\partial i}
\end{gathered}
$$

For this system, the $\mathbf{g}_{0}$ components correspond to the LPE applied to $\bar{R}$ and the $\mathbf{g}_{1}$ components correspond to the LPE applied to $R-\bar{R}$. Therefore, the $\mathbf{g}_{0}$ components are time-invariant and the $\mathbf{g}_{1}$ components are time-periodic. In this case, the time periodicity appears in terms of the true anomaly, $\nu$, with a period of $2 \pi$, which corresponds to one period of the spacecraft about Europa. Because the true anomaly is a function of the mean anomaly, we express the equations as derivatives with respect to mean anomaly rather than with respect to time to simplify the analysis. To transform from time derivatives to derivatives with respect to mean anomaly, the equations must be multiplied by $1 / n$, where $n$ is the mean motion of the spacecraft about Europa.

Because our goal is to determine initial conditions such that the 3DOF trajectory has the 2-DOF trajectory as its average, we linearize about a point in the 2-DOF system. In particular, we linearize about the point in the 2-DOF system that we obtained in the preceding section and let this point be $\mathbf{x}_{0}=\mathbf{x}_{0}^{m}+\delta \mathbf{x}_{0}^{m}$. Recall that in the preceding section, we noted that the terms necessary to solve for the initial conditions, $\mathbf{A}, \mathbf{B}, \mathbf{D}$, and $\mathbf{E}$ were relatively easy to compute. This is not the case for the linearization of the unaveraged system. The constant matrix $\mathbf{A}$ is still straightforward to compute even though it is a $5 \times 5$ matrix. However, the vector $\mathbf{B}$, which in this case is $\mathbf{B}[v(M)]$, cannot be computed analytically because Kepler's equation must be solved to determine the true anomaly, $v$, as a function of the mean anomaly, $M$. Therefore, we turn to the computation of the averages, $\mathbf{D}$ and $\mathbf{E}$. The computation of $\mathbf{D}$ is once again straightforward because $\mathbf{A}$ has already been computed. However, the computation of $\mathbf{E}$ where

$$
\mathbf{E}=\frac{1}{2 \pi} \int_{0}^{2 \pi} \int_{0}^{M} e^{\mathbf{A}(M-\tau)} \mathbf{B}[\nu(\tau)] \mathrm{d} \tau \mathrm{d} M
$$

is more of a challenge. Because it is not possible to compute $\mathbf{B}$ analytically and then average over $M$ to determine $\mathbf{E}$, we use a numerical method to compute the $\mathbf{E}$ directly. In particular, we use the double integral version of Gaussian Quadrature [9] to determine the terms in $\mathbf{E}$. We can then solve for the corrections to $\mathbf{x}_{0}$ and $\delta \mathbf{x}_{0}$.

We now continue with the example from the preceding section to show that when integrated with the initial conditions obtained from the linearized system, the 3-DOF system follows the 2-DOF system on average. The point that we linearize about, which is also the initial condition for the 2-DOF system, is

$$
\mathbf{x}_{0}=\mathbf{x}_{0}^{m}+\delta \mathbf{x}_{0}^{m}=\left[\begin{array}{c}
1682.53057218 \mathrm{~km} \\
0.02027136 \\
70.86067333 \mathrm{deg} \\
171.18752273 \mathrm{deg} \\
0 \mathrm{deg}
\end{array}\right]
$$

The corrections to these initial conditions, $\delta \mathbf{x}_{0}$, are found to be
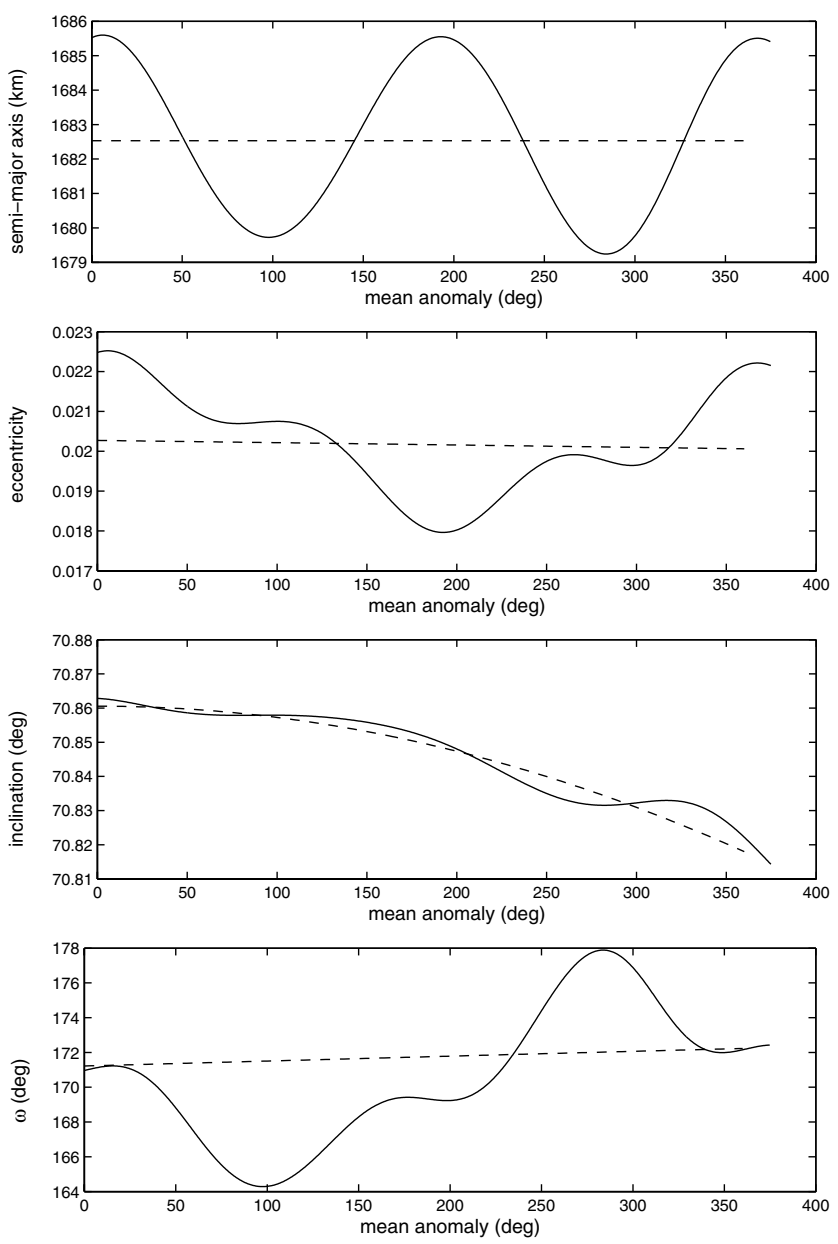

Fig. $15 a, e, i$, and $\omega$ as functions of mean anomaly. Solid curves: integrated 3-DOF trajectory. Dashed curves: integrated 2-DOF trajectory.

$$
\delta \mathbf{x}_{0}=\left[\begin{array}{c}
2.98938278 \mathrm{~km} \\
0.00219834 \\
0.00239633 \mathrm{deg} \\
0.17321674 \mathrm{deg} \\
-0.00679549 \mathrm{deg}
\end{array}\right]
$$

Figure 15 shows plots of the integrated 3-DOF trajectory, initialized at $\mathbf{x}_{0}+\delta \mathbf{x}_{0}$, and the integrated 2-DOF trajectory, initialized at $\mathbf{x}_{0}$. In all four orbital element plots, the 2-DOF trajectory cuts through the center of the 3-DOF trajectory. Therefore, we accomplished our goal of the 3-DOF motion following the 2-DOF motion on average.

\section{Long Lifetime Orbits in the 3-DOF System}

We have now identified an algorithm that starts with a point on the stable manifold of a frozen orbit in the 1-DOF system and produces motion that follows the 1-DOF manifold on average. However, as the trajectory follows the stable manifold towards the frozen orbit (branch labeled II in Fig. 8) there are two possible unstable manifold branches that it could follow (I or III). Because the trajectory has the manifold as its average, there is no guarantee that it will follow the unstable manifold branch corresponding to a long lifetime orbit (I). As an example, consider Fig. 16, which is the numerical integration in the 3 -DOF system of the initial conditions computed in the preceding section.

$$
\mathbf{x}_{0}+\delta \mathbf{x}_{0}=\left[\begin{array}{c}
1685.52995783 \mathrm{~km} \\
0.002246971 \\
70.86306966 \mathrm{deg} \\
171.36073947 \mathrm{deg} \\
-0.00679549 \mathrm{deg}
\end{array}\right]
$$



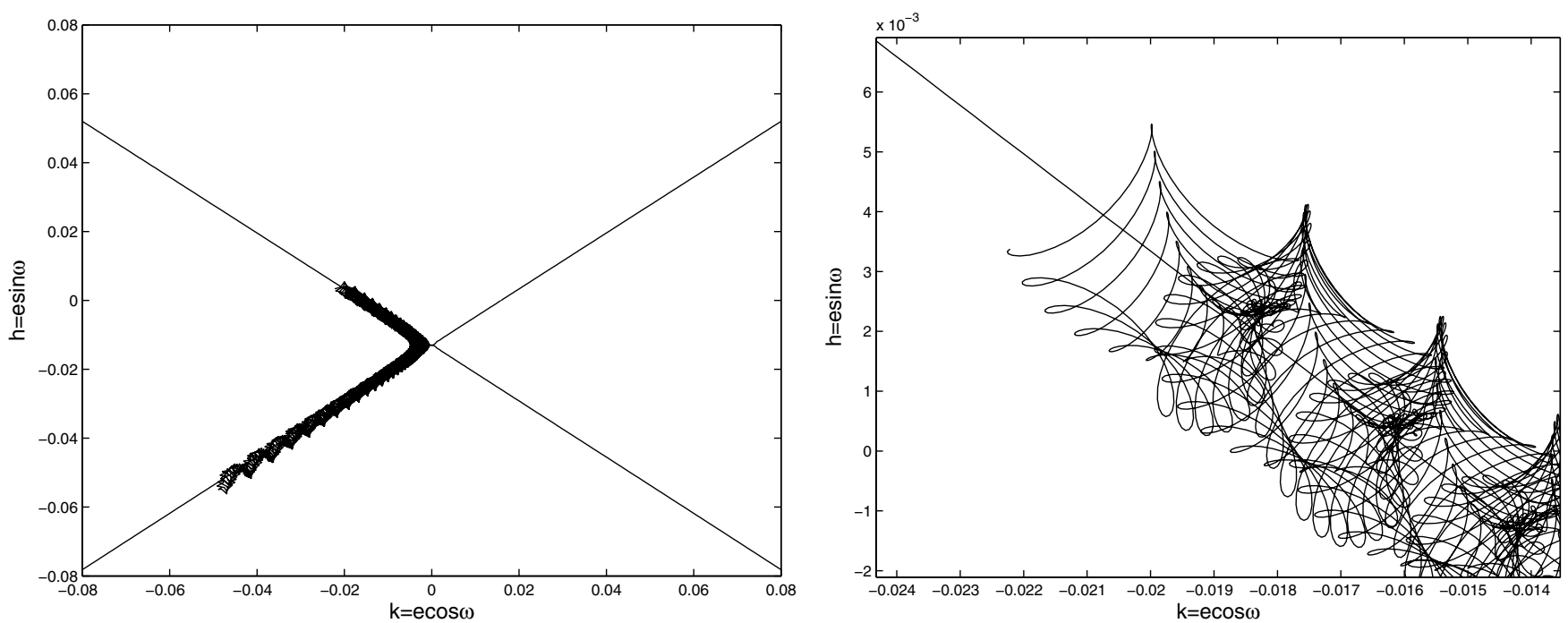

Fig. 16 Left: Integration in 3-DOF system of trajectory initialized at $x_{0}+\delta x_{0}$. Right: Expansion of initial part of trajectory.
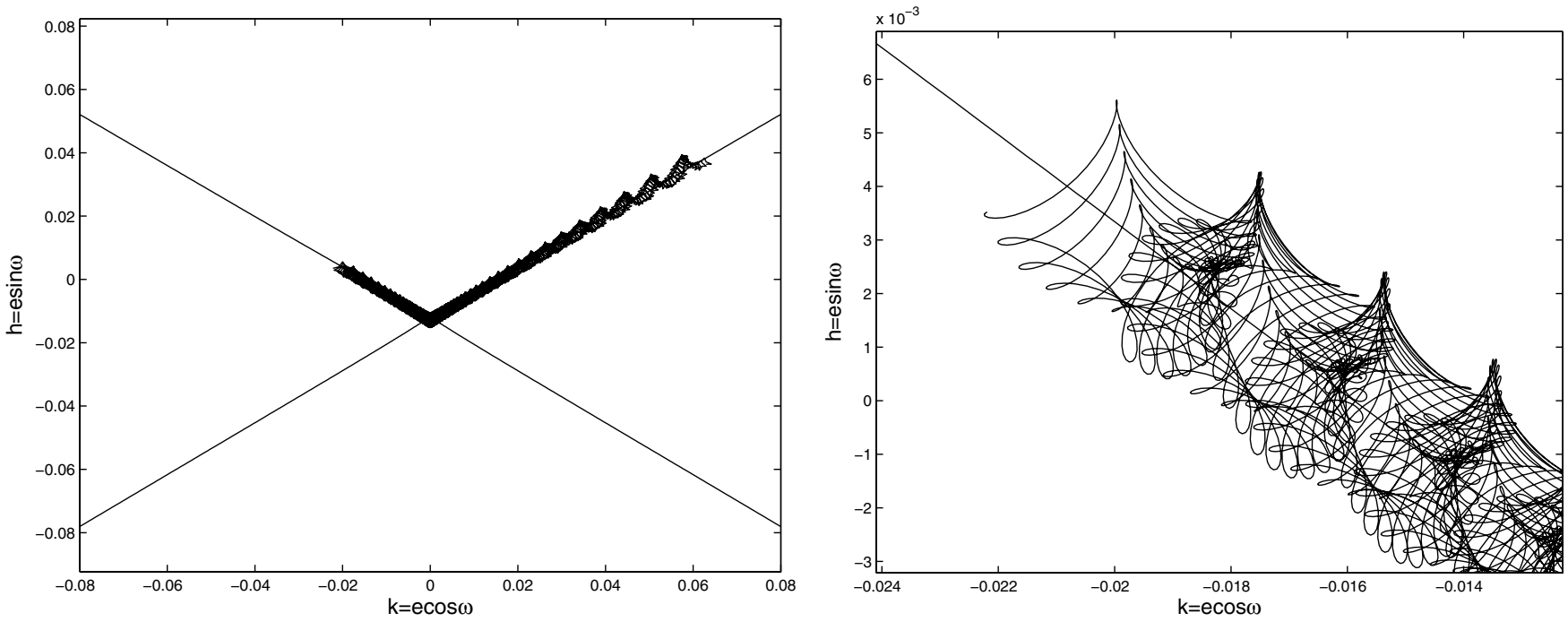

Fig. 17 Left: Integration in 3-DOF system of trajectory initialized with an $\omega$-bias. Right: Expansion of initial part of trajectory.

In Fig. 16 we see that the trajectory does not follow the unstable manifold branch corresponding to a long lifetime orbit. In the zoomed-in portion of the plot on the right, however, we do see that that trajectory does seem to have the 1-DOF manifold as its average. The lifetime of this trajectory is 84 days.

To ensure that the trajectory in the 3-DOF system follows the manifold that leads to a long lifetime orbit, we introduce the concept of a bias. We find that by shifting the argument of periapsis slightly from the computed value, a long lifetime orbit can be obtained. Although at this time we do not have an analytic theory that predicts the necessary shift in argument of periapsis, a numerical search over a small range in argument of periapsis values $(<1 \mathrm{deg})$ can be used to find the initial argument of periapsis that produces the longest lifetime orbit. Therefore, going back to our example, the computed initial argument of periapsis of the orbit that followed the wrong manifold path was $\sim \omega=171.36 \mathrm{deg}$. By introducing a bias of $\sim 0.38 \mathrm{deg}$, we obtain the trajectory shown in Fig. 17 that has a lifetime of 157 days. A summary of the algorithm and the initial conditions obtained at each step for our example is shown in Tables 2 and 3 .

It is important to note that although we have found long lifetime orbits in the 3-DOF system additional considerations must be made when dealing with a real-life system. As we have found, the long lifetime orbits are very sensitive to initial conditions. A small change in the initial argument of periapsis causes the trajectory to follow the wrong manifold branch and reduces the lifetime by half. In reality, we expect that there will be uncertainties in the initial conditions of a true orbit. In general, these uncertainties will be larger than the bias we applied to produce a long lifetime orbit. This means that it will be necessary to make active corrections to ensure that the spacecraft stays on the long lifetime trajectory.

\section{Conclusions}

We have developed an algorithm that is used to design long lifetime trajectories about Europa. Our model includes the tidal force from Jupiter and the $J_{2}, C_{22}$, and $J_{3}$ gravity coefficients from Europa, and is a modified form of the Hill three-body problem. Our analysis begins by reducing the full 3-DOF system by averaging. We perform averaging twice: first over the orbit of the spacecraft about Europa and second over the orbit of Europa about Jupiter. After these two reductions, a simple 1-DOF system that governs the underlying dynamics in the full 3-DOF system is obtained. In the 1-DOF system, frozen orbits with constant values of semimajor axis, eccentricity, inclination, and argument of periapsis on average are identified. One set of frozen orbits contains near-circular, near-polar orbits which are the most suitable for the science orbit of mission a to Europa. However, these orbits are unstable. Manifolds of these orbits in the 1-DOF system are computed, and we show that there is one particular path from the stable to the unstable 
Table 2 Algorithm for finding longest lifetime orbit

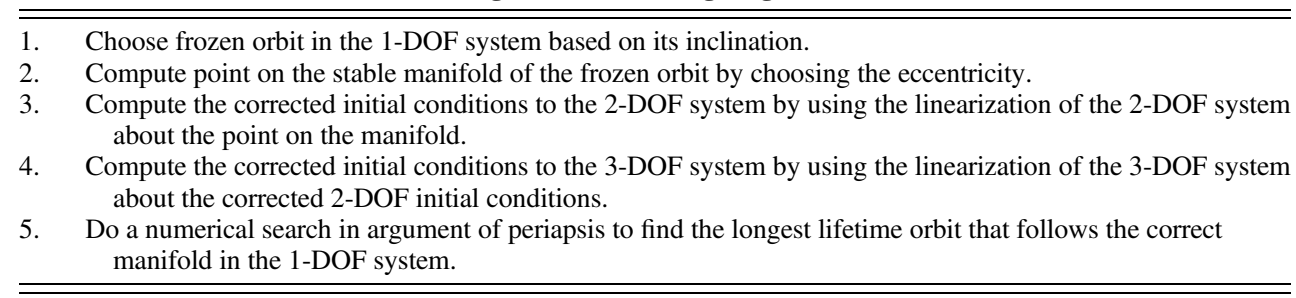

Table 3 Example initial conditions corresponding for the algorithm described in Table 2

\begin{tabular}{lcccrr}
\hline \hline & \multicolumn{5}{c}{ Step in algorithm } \\
\cline { 2 - 6 } & 1 & 2 & 3 & \multicolumn{1}{c}{5} \\
\hline$a, \mathrm{~km}$ & 1682.53057218 & 1682.53057218 & 1682.53057218 & 1685.51995783 & 1685.51995783 \\
$e$ & 0.01291541 & 0.02 & 0.02027136 & 0.02246971 & 0.02246971 \\
$i, \mathrm{deg}$ & 70 & 69.99756766 & 70.86067333 & 70.86306966 & 70.86306966 \\
$\omega, \mathrm{deg}$ & 270 & 170.97339256 & 171.18752273 & 171.36073947 & 171.74175641 \\
$\Omega, \mathrm{deg}$ & N/A & N/A & N/A & -0.00679549 & -0.00679549 \\
$\nu, \mathrm{deg}$ & N/A & N/A & N/A & 0 & 0 \\
\hline \hline
\end{tabular}

manifold that allows the orbit to stay at a low eccentricity while the argument of periapsis circulates. This means that a spacecraft following that path would be on a trajectory with a very long lifetime. The purpose of the algorithm we developed is then to determine initial conditions such that the trajectory in the 3-DOF system follows the manifold in the 1-DOF system on average. The algorithm works by relating the 2-DOF system to the 1-DOF system and the 3-DOF system to the 2-DOF system by linearization. We show that by using the linearizations of both the 2-DOF and 3-DOF systems we can compute initial conditions to use in the 3-DOF system such that the resulting integrated trajectory follows the manifold in the 1-DOF system. We then apply a small correction to the argument of periapsis to ensure that the trajectory in the 3-DOF system follows the correct 1-DOF manifold. The orbits obtained have low eccentricities and near-polar inclinations and have lifetimes on the order of 150 days.

\section{Acknowledgments}

M. E. Paskowitz acknowledges the support of the FXB Foundation. This research was supported by a grant from the Jet Propulsion Laboratory, California Institute of Technology, which is under contract with NASA.

\section{References}

[1] Scheeres, D. J., Guman, M. D., and Villac, B. F., "Stability Analysis of Planetary Satellite Orbiters: Application to the Europa Orbiter," Journal of Guidance, Control, and Dynamics, Vol. 24, No. 4, 2001, pp. 778-787.

[2] Broucke, R. A., "Long-Term Third-Body Effects via Double Averaging," Journal of Guidance, Control, and Dynamics, Vol. 26, No. 1, 2003, pp. 27-32.

[3] San-Juan, J. F., Lara, M., and Ferrer, S., "Phase Space Structure Around Planetary Satellites," AIAA Paper 2004-4863, 2004.

[4] Lara, M., San-Juan, J. F., and Ferrer, S., "Secular Motion Around TriAxial, Sychronously Rotating Planetary Satellites: Application to Europa," Advances in the Astronautical Sciences, Vol. 120, No. 2, 2005, pp. 1147-1166.

[5] Brouwer, D., and Clemence, G. M., Methods of Celestial Mechanics, Academic Press, New York, 1961, pp. 290, 337.

[6] Montenbruck, O., and Gill, E., Satellite Orbits: Models, Methods, and Applications, Springer-Verlag, Berlin, 2001, pp. 30, 57.

[7] Chobotov, V. A., (ed.), Orbital Mechanics, 2nd ed., AIAA, Reston, VA, 1996, p. 201

[8] Paskowitz, M. E., and Scheeres, D. J., "Orbit Mechanics About Planetary Satellites Including Higher Order Gravity Fields," Advances in the Astronautical Sciences, Vol. 120, No. 2, 2005, pp. 1443-1462.

[9] Burden, R., and Faires, J., Numerical Analysis, 6th ed., Brooks/Cole Publishing Company, Pacific Grove, CA, 1997, p. 202. 